\title{
Cosmic rays and hadronic interactions
}

\author{
Paolo Lipari ${ }^{\mathrm{a}}$ \\ INFN sezione Roma Sapienza
}

\begin{abstract}
The study of cosmic rays, and more in general of the "high energy universe" is at the moment a vibrant field that, thanks to the observations by several innovative detectors for relativistic charged particles, gamma-rays, and neutrinos continue to generate surprising and exciting results. The progress in the field is rapid but many fundamental problems remain open. There is an intimate relation between the study of the high energy universe and the study of the properties of hadronic interactions. High energy cosmic rays can only be studied detecting the showers they generate in the atmosphere, and for the interpretation of the data one needs an accurate modeling of the collisions between hadrons. Also the study of cosmic rays inside their sources and in the Galaxy requires a precise description of hadronic interactions. A program of experimental studies at the LHC and at lower energy, designed to address the most pressing problems, could significantly reduce the existing uncertainties and is very desirable. Such an experimental program would also have a strong intrinsic scientific interest, allowing the broadening and deepening of our understanding of Quantum Chromo Dynamics in the non-perturbative regime, the least understood sector of the Standard Model of particle physics. It should also be noted that the cosmic ray spectrum extends to particles with energy $E \sim 10^{20} \mathrm{eV}$, or a nucleon-nucleon c.m. energy $\sqrt{s} \simeq 430 \mathrm{TeV}, 30$ times higher than the current LHC energy. Cosmic ray experiments therefore offer the possibility to perform studies on the properties of hadronic interactions that are impossible at accelerators.
\end{abstract}

\section{Introduction}

Approximately one hundred years ago, the observations of Victor Hess and other pioneers lead to the discovery of the existence of cosmic rays. This discovery provided the first, completely unexpected, glimpse of the "High Energy Universe", revealing the existence of astrophysical sources and mechanisms, that somehow are capable of accelerating particles to extraordinary high energies.

Cosmic Rays (CR) played a crucial role in the birth of "Particle Physics". The primary CR particles, and the secondaries generated by their interactions with the air nuclei, were used to study the properties of fundamental interactions in new conditions, and Particle Physics was born from the discovery of new elementary particles (the positron, the muon, the charged and neutral pions, the kaons and the Lambda), and the study of their properties.

In the 1950's the experimental studies in Particle Physics made a transition from the use of the fluxes of cosmic rays particles, freely provided by Nature, to the use of more intense and better controled accelerator beams. A remarkable story of experimental and theoretical efforts has culminated in the construction and verification of the "Standard Model", a quantum field theory based on the gauge symmetry of the $S U(3) \otimes S U(2) \otimes U(1)$ group. The last missing element of the theory, the Englert-BroutHiggs boson, has been recently discovered at LHC.

In contrast to the rapid development of particle physics, the study of the origin of cosmic rays and our understanding of the "High Energy Universe" for several decades has progressed only slowly. The main difficulty

$\overline{\text { a e-mail: Paolo.Lipari@roma1.infn.it }}$ in addressing this problem follows from the fact that the trajectories of relativistic charged particles in our Galaxy and in intergalactic space, are bent by magnetic fields, so the observed directions of CR particles do not point to the sources that remain unidentified.

The observed spectra are formed by the superposition of the contributions of many different sources that were active (each probably for a short time interval) at different epochs, and are shaped not only by the properties of the sources that inject the particles in space, but also by the mechanisms of propagation in galactic or extragalactic space.

The situation has dramatically changed in the last 25 years, when the field of High Energy Astrophysics has made impressive progress. The most important development has been the development of new telescopes for gamma astronomy in the energy range from (approximately) $100 \mathrm{MeV}$ to $100 \mathrm{TeV}$. Photon emission in this energy range is due to the interactions of relativistic charged particles, and maps the astrophysical sites where cosmic rays are created or stored. The new instruments, placed in space (Egret, Agile, Fermi) and on the ground (Whipple, Hess, Magic, Veritas, Milagro, ARGO-YBJ), have identified many sources, proving that the acceleration of particles to relativistic energy is a phenomenon that exists in several classes of astrophysical objects (such as Super Nova Remnants, Gamma Ray Bursts, Pulsar Wind Nebulae, Microquasars, Active Galactic Nuclei, ...), and is associated to different mechanisms. Both electrons and positrons and protons and nuclei can be the source of high energy photons. In the case of $e^{\mp}$ the emission mechanism is Compton scattering or bremsstrahlung; for protons and nuclei the source of the gamma rays is the decay of 
neutral pions and other mesons ( $\operatorname{such}$ as $\eta$ and $\eta^{\prime}$ ) produced in hadronic interactions of the particles with matter or radiation fields.

The interactions of relativistic hadrons are also a source of neutrinos that are produced in the (chain) decay of mesons created in the inelastic interactions (such as $\pi^{+} \rightarrow \mu^{+} v_{\mu}$, followed by $\mu^{+} \rightarrow e^{+} \nu_{e} \bar{v}_{\mu}$ and charge conjugate modes). Very large efforts have been invested in the design and construction of telescopes for high energy neutrino astronomy. The first observation ${ }^{1}$ of high energy astrophysical v's above the foreground of atmospheric neutrinos, have been finally obtained by IceCube, with a signal emerging at very high energy $E_{v} \gtrsim 100 \mathrm{TeV}$.

The experimental study of charged cosmic rays has also made important progress in recent years thanks to the data collected by new detectors in space (Pamela, AMS), on balloons (ATIC, CREAM) and at ground level. These new measurements have measured with unprecedented precision the energy spectra of cosmic rays in a very broad energy range that covers eleven or more decades, from a kinetic energy of approximately $100 \mathrm{MeV}$, to a maximum energy of order $10^{20} \mathrm{eV}$. The observations have revealed the existence of subtle but clear spectral features that do not yet have a clear and universally accepted explanation and are currently the object of a lively scientific debate.

The topic that has attracted the most attention in the discussion about recent CR observations is the shape and properties of the spectra of anti-particles (positrons and anti-protons). The expectation is that the dominant source of relativistic $e^{+}$and $\bar{p}$ is the interaction with interstellar gas of CR protons and nuclei confined by magnetic fields inside our Galaxy. A flux larger than the predictions based on this assumption could be the indication of a new source of relativistic anti-particles, perhaps due to the annihilation of Dark Matter particles in the form of Weakly Interacting Massive Particles (WIMP's), or alternatively would require a profound revision of our understanding of CR propagation.

The three fields of cosmic rays, gamma and neutrino astronomy have now merged in a single one that can be called "Particle Astrophysics", directed at the discovery, classification and understanding of the sources and mechanisms that generate high energy particles. This field at the moment is remarkably rich and vibrant, with new observations that continuously provide surprising and exciting results that challenge the theorists, and motivate the efforts for the design of new instruments that can perform the observations needed to address the open problems.

During these decades of efforts the two fields of "Particle" and "Astroparticle" physics have remained closely related. As already mentioned, cosmic rays were of fundamental importance in the early years of discovery of particle physics. Later, in the "accelerator era" very

\footnotetext{
${ }^{1}$ We should remember here that at much lower energy extraterrestrial neutrinos have already been observed from the Sun and SuperNova SN1987A. New observations of SuperNova neutrinos (if and when ...) have great interest, and the potential to give very valuable information about the SN explosion mechanism, and the fundamental neutrino properties. Note that hadronic physics, is a key element of the problem.
}

valuable information mostly flowed in the opposite direction, for example when the energy dependence of hadronic cross section measured in experiments performed at the hadronic colliders are included to obtain a more accurate interpretation of the cosmic ray observations. In fact, also today, and in the foreseeable future, the experimental studies of CR particles at high energy are performed observing the showers they generate in the atmosphere, and a precise modeling of the shower development is required to correctly interpret the measurements. Uncertainties on the properties of hadronic interactions are the dominant source of systematic error in these studies, and are an important limitation. Appropriate programs of experimental studies at the LHC and also at lower energy could reduce these uncertainties and greatly benefit the field.

The science at the intersection of the two fields of Particle and Astroparticle physics, that is the subjects of the series of ISVHECRI (International Symposia on Very High Energy Cosmic Ray Interactions), remains today a field rich, complex and of great interest.

\section{QCD: The "dark side" of the standard model}

There are very strong arguments to believe that the underlying theory that describes the interactions between hadronic particles is Quantum Chromo Dynamics (QCD), one of the fundamental pieces of the Standard Model of Particle Physics. The fundamental fields in the theory are quarks (spin 1/2 fermions) and gluons (massless spin 1 gauge bosons), while the physical hadrons are bound states composed of three quarks in the case of baryons (three anti-quarks in the case of anti-baryons), or a quark and anti-quark in the case of mesons.

The main steps in establishing the validity of QCD are well known. The finite size of the proton, and its radius of order $1 \mathrm{fm}$, were established in electron scattering experiments performed in the 1950's by Robert Hofstadter. Approximately ten years later, new electron scattering experiments performed at higher energy by Jerome Friedman, Henry Kendall and Richard Taylor demonstrated that the proton contains an ensemble of point-like, electrically charged entities that were soon identified with quarks, the fractionally charged, "fundamental entities" postulated by Gell Mann to explain the properties and symmetries of baryon and meson particles. A few years later D. Gross, D. Politzer and F. Wilczek established that the quantum field theory based of the gauge symmetry of the SU(3) group, or Quantum Chromodynamics (QCD) has the property of "asymptotic freedom", so that the fundamental fields of the theory (quarks and gluons) appear almost free when looked at with high resolution probes.

At the other extreme, QCD theory implies "confinement". The force between the fundamental fields does not decrease with distance, and the isolation of a quark or a gluon requires an infinite amount of energy, and is therefore impossible. A formal, rigorous proof that QCD (or in more general gauge theories) has confinement (or a "mass gap") does not yet exist, and finding such a 
proof is one of the "Millenium problems" announced by the Clay Mathematics Institute, with a million dollar prize offered for a solution. On the other hand, taking a more "phenomenological" approach, few physicist have any doubt that quarks and gluons are confined, and that only colorless combinations are physically observable.

From the combination of asymptotic freedom and confinement emerges a remarkable situation for hadronic interactions. It is possible to compute with very high precision "hard" (high $Q^{2}$ ) processes such as electron scattering at large momentum transfer, or Higgs production at the LHC, but there is no reliable method to compute "soft" (low $Q^{2}$ ) processes. For the calculation of hard processes one needs to have some knowledge of the structure of the hadrons that participate in a collision. This information is encoded in the Parton Distribution Functions (PDF's) $F_{a}^{(h)}\left(x, Q^{2}\right)$ that give the probability of finding inside hadron $h$ the parton type $a$ with fractional momentum $x$ in a process with momentum transfer $Q^{2}$. The $Q^{2}$ evolution of the PDF's is calculable in QCD, but only starting from initial conditions that are determined experimentally and have a dependence from the momentum transfer $Q^{2}$ that is calculable. Convoluting the PDF's with elementary lepton-parton or parton-parton cross sections it is possible to calculated in perturbation theory, and with high accuracy, the properties of hard processes such as a high $p_{\perp}$ jet-pair, or the production of a Higgs boson.

Soft processes account for most of the cross section in hadronic interactions, and determine the total, elastic and inelastic cross sections and the main properties of the final state: the multiplicity distribution, the composition (relative abundance of different particle types), the inclusive particle spectra and so on. We do not have viable methods to calculate from first principles these properties of hadronic interactions, and when this information is needed, one has to rely on experimental measurements.

\section{Hadronic interactions and high energy astrophysics}

A detailed knowledge of the properties of hadronic interactions is very important for cosmic ray and more in general high energy astrophysics studies. The connection has several motivations. The first, and perhaps most important one, is the need for a precise description of the development of hadronic showers. The flux of high energy cosmic rays is so small that they can only be studied with indirect methods, observing the showers generated when they interact in the Earth's atmosphere. The problem then is to estimate the energy and identity (the mass number $A$ in the case of nuclei) of the primary particles from the measurements of shower observables. This task requires an accurate model for the development of hadronic showers. The main source of systematic uncertainties for this task is due to our limited understanding of the properties of hadronic interactions.

A precise description of hadronic interaction is also important for the interpretation of other cosmic ray and gamma ray observations that have significant implications for the field of high energy astrophysics. Cosmic ray interactions with the low density gas that fills interstellar space are the source of secondary particles, including rare nuclei formed by the fragmentation of higher mass primaries (in processes such as ${ }^{12} \mathrm{C}+p \rightarrow{ }^{10} \mathrm{Be}+3 p$ ), positrons and antiprotons. The main source of positrons is the chain decay of charged pions and other mesons (such as $\pi^{+} \rightarrow \mu^{+} \rightarrow e^{+}$), while antiprotons are created in reactions such as $p p \rightarrow \bar{p} p p p+\ldots)$. The crucial point is that the injection of secondary particles in interstellar space can be calculated folding the energy spectra of primary particles with the appropriate inclusive cross sections. The experimental study of the fluxes of these secondary particles is of great importance in high energy astrophysics because a comparison of the measured fluxes with the calculated injection rates allows to obtain information on the properties of cosmic ray propagation in the Galaxy, such as the average residence time for charged particles of different energy. A precise knowledge of the relevant cross sections is essential.

An additional and compelling motivation for the study of the fluxes of positrons and antiprotons is that if the galactic Dark Matter is in the form of Weakly Interacting Massive Particles (WIMPs), the self-annihilation or decay of these particles can be a source of $e^{+}$and $\bar{p}$ (the production of secondary particles in DM annihilation or decay is expected to be symmetric under charge conjugation, but the production of $e^{-}$and $p$ will be hidden under a much larger background). To disentangle a DM signal from the expected background clearly requires an accurate calculation of the spectrum and normalization for the fluxes of $e^{+}$and $\bar{p}$ from known conventional sources, and this reinforces the importance of a good understanding of the relevant hadronic physics.

One of the crucial goals of $\gamma$-ray astronomy is the determination of the size and energy spectrum of the populations of relativistic particles (electrons, protons and nuclei) present in different astrophysical sources. The shape of the spectrum of the $\gamma$-ray emission generated by a population of relativistic protons in an astrophysical source is determined by the convolution of their energy distribution with the differential cross section for the production of neutral pions in their collisions with the source material $\left(\pi^{\circ}\right.$ are the main source of gamma rays via the decay $\pi^{\circ} \rightarrow \gamma \gamma$ ). This mechanism of photon production has a characteristic signature because, for simple kinematical reasons, the emission spectrum has a maximum at $E_{\gamma} \simeq m_{\pi^{0}} / 2$. The observation of this so called "pion bump" is an important indication of the presence of protons in an astrophysical source, and has been recently observed by Agile and Fermi $[1,2]$.

The detailed shape of the photon spectrum around $E_{\gamma} \simeq m_{\pi^{0}} / 2$ gives interesting information on the energy distribution of the protons in the source, but uncertainties in the description of pion production in $p p$ interactions at low energy $\left(E_{p} \sim\right.$ few $\mathrm{GeV}$ ) remains a non negligible source of error in the interpretation of the observations.

An additional situation where a detailed knowledge of hadronic interactions is of interest for high energy astrophysics is the estimate of the contribution to the atmospheric neutrino flux due to the production and decay of hadrons that contain a charm quark. As discussed in the 
following, this flux is an important source of foreground for the determination of the diffuse flux of astrophysical neutrinos. In this case the problem is to estimate the inclusive cross sections for the production of charm mesons and baryons. This problem is at the border between what can be reliably calculated in perturbation theory, and the non-perturbative regime. For more discussion see [3].

In the absence of a reliable method to calculate soft hadronic processes one needs to use data obtained in accelerator experiments to determine experimentally the properties of hadronic interaction that are required for different scientific objectives.

In recent years (in fact during several decades) a lot of very valuable data has been collected in many experiments performed at different accelerators. These data, together with a theoretical framework to interpolate and extrapolate the experimental results are a fundamental ingredient in many studies in cosmic rays and high energy astrophysics.

It should however be stressed that a program of experimental studies directed toward the most critical open questions would be of great interest for cosmic ray studies, and is therefore very desirable.

The most important "frontier" for these studies is at high center of mass energy. The CR spectrum extends (at least) to $E \simeq 10^{20} \mathrm{eV}$, that corresponds for proton primaries to a c.m. energy for nucleon-nucleon interactions $\sqrt{s} \simeq 433 \mathrm{TeV}$, significantly higher than what has been studied in accelerator experiments. The data collected at the LHC collider at CERN are therefore of crucial importance, significanly reducing the extrapolation needed to model the showers of the highest energy cosmic rays.

The particles in the final state of a hadronic interaction that play the most important role in the development of CR showers are those that carry a large fraction of the projectile particle energy, and penetrate deeper in the atmosphere. The so called "projectile fragmentation region" is, however, the kinematical region where the measurements in collider experiments are most difficult, because the particles have very small angles with respect to the accelerator beams. Efforts for accurate studies in the very forward region at LHC experiments, hopefully also going beyond the present detectors, are clearly very important.

One should of course also remember that CR showers develop in air, where the targets are nuclei (nitrogen, oxygen and argon), and that a significant part of the CR are nuclei. The interpretation of $\mathrm{CR}$ observations requires then the modeling of hadron-nucleus and nucleus-nucleus interactions, while much of the data available at the highest energies have been obtained in hadron-hadron collisions. For example, we now have measurements of the $p p$ total and elastic cross section up to $\sqrt{s}=8 \mathrm{TeV}$, but the protonnucleus and nucleus-nucleus cross sections at the same c.m. energy can only be calculated (using extensions of the Glauber theory), introducing theoretical uncertainties. For this reason the experimental programs for proton-nucleus and nucleus-nucleus collisions at RHIC and LHC have been, and will be of great interest for the CR community.

The relation between cosmic rays and hadronic physics can be rich and complex. The results of accelerator

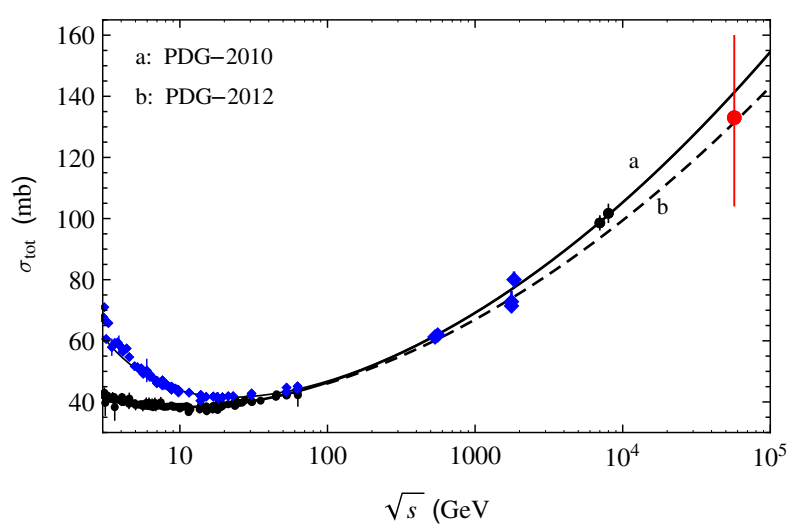

Figure 1. Total $p p$ and $\bar{p} p$ cross section. The lines are the predictions of PDG-2010 [5] and PDG-2012. The point at $\sqrt{s}=57 \mathrm{TeV}$ was estimated bu the Auger collaboration from CR observations [7].

experiments on hadronic collisions (and the theoretical frameworks constructed for their interpretation) are used to understand the nature of the CR fluxes, but the results of CR observations can also be used to obtain information on hadronic interactions in regions of kinematical space that are not accessible with accelerators. This task can appear impossible, because the properties of the CR beam are not known a priori, and have to be determined experimentally, from the same observations used to study hadronic interactions. However, the requirement of a consistent interpretation for a well chosen set of observations can in principle break the apparent circularity of the problem.

\section{High energy hadronic interactions}

In QCD, protons and neutrons are complex and spatially extended objects formed by many elementary objects. The spatial extension of the nucleons can be studied in electron scattering experiments. The proton electromagnetic form factor is well described by the expression $F\left(Q^{2}\right) \simeq[1+$ $\left.r_{0}^{2} Q^{2}\right]^{-2}$ with $r_{0} \simeq 0.234 \mathrm{fm}$. This corresponds (after performing a Fourier transform) to an electric charge density distribution that is approximately exponential $\left(\rho(r) \propto e^{-r / r_{0}}\right)$. Electron (and neutrino) scattering at larger $Q^{2}$ (smaller distances) reveals how the electric charge is associated to point-like partons (the quarks). The order of magnitude of the $p p$ hadronic cross section is in fact determined by the proton spatial extension $\left(\sigma_{p p} \sim\right.$ $\pi\left(2 r_{0}\right)^{2} \simeq 28$ mbarn). The $p p$ cross section grows slowly with c.m. energy as illustrated in Fig. 1, that include the measurements obtained at the LHC by the TOTEM [4] detector for $\sqrt{s}=7$ and $8 \mathrm{TeV}$, and the estimate obtained from CR observations by the Auger collaboration at $\sqrt{s}=$ $57 \mathrm{TeV}$ (see discussion below).

The lines in the figure are functional fits to the $p p$ cross section from the 2010 [5] and 2012 [6] versions of the Particle Data Group review. In this parametrization the cross section grows $\propto \ln ^{2} s$ (the same energy dependence of the so called "Froissart bound"). The question if there is a robust theoretical understanding behind this result remains controversial (see for example the discussion in [8]). 
The growth with energy of $\sigma_{\text {tot }}(s)$ can be interpreted qualitatively as the consequence of the fact that the number of parton-parton interactions increases when the kinematical space expands. This idea implies that the growth of the cross sections should also be accompanied by a more complex structure of the final states due to the presence of multiple parton interactions in the same collision.

In essentially all existing models, multiple interactions in hadron collisions are included in the theoretical framework using an eikonal formalism. The elastic scattering amplitude (that gives also the total cross section via the optical theorem) is written as an integral in impact parameter space:

$f_{\mathrm{el}}(q, s)=i \int \frac{d^{2} b}{2 \pi} e^{i \vec{q} \cdot \vec{b}} \Gamma(b, s)=i \int \frac{d^{2} b}{2 \pi}\left(1-e^{i \chi(b, s)}\right)$

where $\vec{q}$ is the transfer momentum (approximated as a purely transverse spatial vector), $\Gamma(b, s)$ is the profile function, and $\chi(b, s)$ the eikonal function.

The theory attempts to connect the eikonal function to the parton-parton cross section. In the simplest models one writes

$$
\chi(b, s) \simeq \frac{1}{2} \sigma_{\text {parton }}(s) A(b, s)
$$

where $\sigma_{\text {parton }}(s)$ is the total cross section for parton interactions (that has contributions from both hard and soft interactions) and $A(b, s)$ is a profile function $A(b, s)$ that describes the overlap of the spatial (impact parameter space) distributions of hadronic matter in the colliding protons. Note that Eq. (2) implies that the ratio $\sigma_{\text {parton }}(s) / \sigma_{\text {inel }}(s)$ gives the average number of elementary interactions per inelastic collision.

A discussion of the problems associated to the definition of a parton cross section, and the meaning of an elementary soft interaction is beyond the scope of this brief review. Many questions remain very controversial in what is sometimes called "Gribov-Regge multiple scattering theory" [9]. Also very controversial from the theory point of view is the role of the pomeron (or pomerons) in hadronic physics, that for some is an important theoretical object, and for others is a misleading, ill-defined or perhaps even meaningless concept (for a discussion see [10]).

The structure of the final state of inelastic hadron interactions (particle composition, multiplicity, energy spectra, fluctuations and so on) is of great importance for shower development (see for example [11]). If the particles of the final state have on average large (small) energy the showers are more (less) penetrating. For this reason it is important to describe correctly the relative importance of the diffractive and non-diffractive components in the inelastic cross sections that accounts for approximately $20 \%(80 \%)$ of the inelastic cross sections. Diffractive interactions can be seen as a process where one (or both) of the colliding protons is (are) excited into a state with the same internal quantum numbers of the initial particle; the beam and projectile particles then undergo an elastic scattering with small momentum transfer. Accordingly the diffractive cross section can be naturally divided into three components: target, beam and double diffraction. The mass distribution of the excited state peaks at low masses (with the $\Delta$ resonance with $m_{\Delta} \simeq 1.232 \mathrm{GeV}$ playing a prominent role). Single diffraction events in $p p$ collisions have therefore one proton that carries an energy close to the initial state particle in one hemisphere, and a small number of high energy particles (formed by the decay of the excited nucleon resonance) in the opposite hemisphere. In double diffraction events one has two sets of particles produced in the decay of two resonances that are separated by a "rapidity gap".

From the point of view of theory, the problem is to predict the diffractive cross section in a consistent way. This can, in principle, be obtained in the multichannel eikonal model (see for example the discussions in [12-14]).

In non-diffractive events nearly all the secondary particles are produced with a small transverse momentum (with respect to the direction collinear with the momenta of the initial state particles) and a broad distribution in longitudinal momentum. The $p_{\perp}$ distribution is approximately gaussian, with an average of order $0.3-0.5 \mathrm{GeV}$ (that depends on particle type and c.m. energy). The $p_{\perp}$ distribution has a tail of power law form that becomes more important with increasing $\sqrt{s}$, which can be understood as the result of the increasing importance of hard scattering between partons.

The longitudinal momentum distribution can be best described in the c.m. frame, where most of the secondaries are soft. The inclusive spectra for mesons are strongly suppressed when the longitudinal momentum is close to the kinematical limits $\left(p_{z, \max } \simeq \pm \sqrt{s} / 2\right)$. In the case of nucleons one finds one "leading" nucleon per hemisphere with large momentum and a distribution that extends to the kinematical limit.

In 1969 Feynman, extrapolating the first observations of the experimental results available at the time, suggested [15] that the inclusive differential energy spectra of the final state particles satisfy asymptotically (at large $\sqrt{s}$ ) a scaling property, now known as "Feynman scaling":

$$
\frac{d N_{j}}{d p_{z}}\left(p_{z}, \sqrt{s}\right) \simeq \frac{1}{E} F_{j}\left(\frac{2 p_{z}^{*}}{\sqrt{s}}\right)=\frac{1}{E} F_{j}\left(x_{F}\right) .
$$

In the equation above $N_{j}$ is the number of particles of type $j$ produced in the interaction (and $d p_{z} / E$ is a relativistic invariant). The important point is that the function $F_{j}$ depends only on the ratio $x_{F}=2 p_{z}^{*} / \sqrt{s}$ (or "X-Feynman", with $p_{z}^{*}$ the momentum in the c.m. frame) for all values of the c.m. energy $\sqrt{s}$. When $x_{f} \rightarrow 0$, the function $F_{j}\left(x_{F}\right)$ goes to a finite value, and the inclusive spectra takes approximately the form $d N_{j} / d p_{z} \propto 1 / E$. This can be restated by saying that the rapidity distribution $d N_{j} / d y$ in the central region ( $y=0$ in the c.m. frame) is flat with a constant $(\sqrt{s}$ independent $)$ value. The rapidity $y$ is defined by the differential equation: $d y=d p_{z} / E$ that can be integrated to obtain (for the boundary condition $y=0$ when $\left.p_{z}=0\right): y=1 / 2 \log \left[\left(E+p_{z}\right) /\left(E-p_{z}\right)\right]$.

Feynman scaling is not an exact property of the spectra in the final states of hadron-hadron collisions, however it is an important concept for hadronic interactions, because it is a property of the hadronization of certain parton 


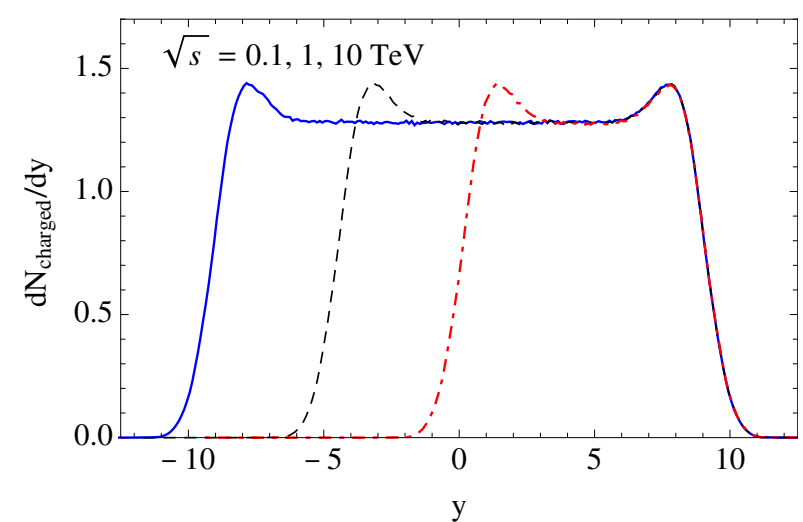

Figure 2. Example of the rapidity distributions of particles obtained from the fragmentation of a $u \bar{u}$ string, calculated with Monte Carlo methods using the LUND algorithm t[18] for three values of the mass of the system. The distributions for the highest mass is shown in the c.m. system, in the other cases the distribution is boosted to have the same $y_{\max }$.

sytems with identical structure but different mass. For example, a $q \bar{q}$ system created in $e^{+} e^{-}$annihilation at $\sqrt{s}=M$ fragments into a set of hadrons having spectra that are experimentally consistent with Feynman scaling, and the iterative algorithms for the fragmentation of such a $q \bar{q}$ system developed following the Field and Feynman ansatz [16] and now in common use in different Monte Carlo codes (such as those of the Lund group [17]). An illustration of this scaling behaviour is shown in Fig. 2.

Assuming the validity of Feynman scaling it would be straightforward to extrapolate the existing accelerator data to an arbitrary energy predicting shower development for CR of an arbitrary primary energy. On the other hand the scaling is violated in high energy hadron collisions. The clearest evidence for the existence of scaling violations is the measurement of the charged particle rapidity distributions for collisions at different $\sqrt{s}$. In fact it is simpler to measure the distribution in the variable pseudo-rapidity $\eta=1 / 2 \ln \left[\left(p_{l}+p_{\perp}\right) /\right.$ $\left.\left(p_{l}-p_{\perp}\right)\right]=1 / 2 \ln [(1+\cos \theta) /(1-\cos \theta)]$. The pseudorapidity $\eta$ depends only on the (easily measured) direction of the particle, and is identical to the rapidity $y$ in the case of massless particles. The particle density $d N / d \eta$ for $\eta \simeq 0$ grows with $\sqrt{s}$ as shown in Fig. 3 (note that a flat distribution for $y$ corresponds to an $\eta$ that has minimum at $\eta \simeq 0$ because of the Jacobian factor in the variable transformation).

Because of energy conservation, the increase in the particle density in the central region corresponds to a softening of the spectra in the two fragmentation regions, that is a suppression of the production of particles at large $\left|x_{F}\right|$. This effect is phenomenologically very important for CRs. In fact, looking at the collision in the laboratory frame, particles in the backward hemisphere $\left(x_{F}<0\right)$ have low energy (of order $E_{\mathrm{lab}} \simeq \sqrt{m^{2}+p_{\perp}^{2}}$ ), but for particles in the forward hemisphere $\left(x_{F}>0\right)$ one has $E_{\text {lab }} \simeq E_{0} x_{f}$ (with $E_{0}$ the primary energy), and $x_{F}$ distribution can then be simply interpreted as the laboratory energy distribution.

As already discussed, some of the properties of hadronic interactions that are most significant for the

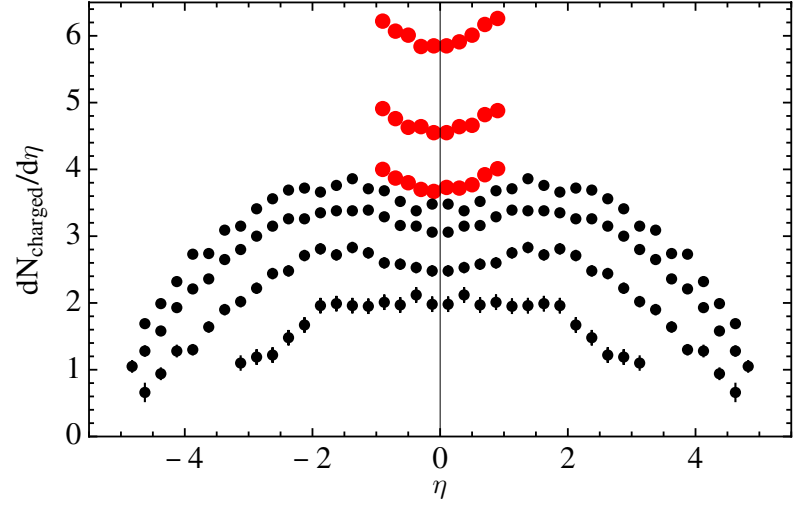

Figure 3. Pseudorapidity distributions measured in $\bar{p} p$ interactions by the UA 5 experiment at the CERN S $\bar{p} p \mathrm{~S}$ collider at $\sqrt{s}=$ $53,200,546$ and $900 \mathrm{GeV}$, and in $p p$ interactions by ALICE at the LHC at $\sqrt{s}=0.9,2.36$ and $7 \mathrm{TeV}$.

development of CR showers, such as the energy distribution of the leading nucleon, and more in general of particles at large $\left|x_{F}\right|$ are very difficult to measure in collider experiments, because these particles are emitted at very small angles with respect to the beam directions. Special detectors such as LHCf have been operating to study the fastest secondary particles in collisions at LHC. The LHCf results Fig. 4 [19-21] show interesting discrepancies with the available Monte Carlo results. The implication of these results for CR shower development are still not clear, because the data were obtained in a very restricted kinematical region, and the determination of the inclusive energy spectra depends on assumptions about the $p_{\perp}$ distributions. A more complete coverage of $p_{\perp}$ for high energy secondaries could resolve these ambiguities and would be very beneficial.

Several Monte Carlo models have been constructed for the generation of $p p$ collisions. Some of these codes such as PYTHIA, HERWIG or SHERPA [22-25] have as primary goal the interpretation of the accelerator collisions, with the main focus on rare hard processes, including manifestations of possible new physics. Also in this case it is, however, necessary to address the problem of the properties of particles produced in soft interactions (the so called "underlying event" in the collisions where a hard process has been identified) [26].

In most cases the codes developed for experiments at accelerators cannot be easily used for CR studies, because they lack the possibility to model the interactions of mesons, or nuclei, or change easily the c.m. energy of the interactions during a single computer run, as required for shower simulations. Because of these limitations other Monte Carlo models such as QGSJET, SIBYLL or EPOS [27-30] are in common use for the simulations of CR showers. For more discussion see [31].

The models used at accelerators have versions with the parameters "tuned" to be in agreement with the data obtained at a fixed $\sqrt{s}$. The codes used for CR studies (that neglect the rare processes of highest interest in particle physics) optimize their parameters to reproduce the data in a very broad range of $\sqrt{s}$ because showers contain particles of all energies below the energy of 

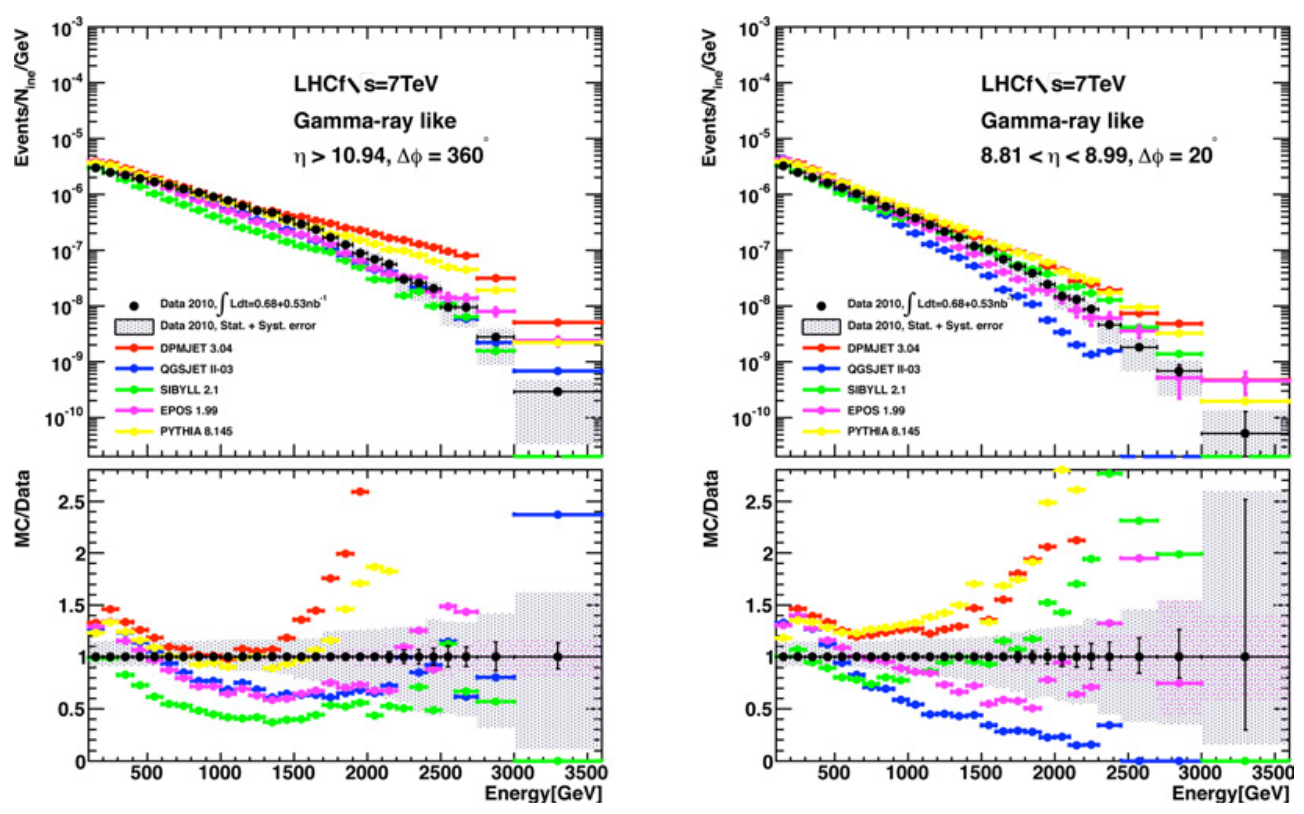

Figure 4. Data of the LHCf detector at $7 \mathrm{TeV}$ [19].

the primary. In addition the codes for CR studies at the highest energies need to consider the extrapolation beyond the range accessible at colliders, which can be an important constraint in choosing the energy dependence of the model parameters. The Monte Carlo models are reasonably successful in describing the LHC results, but some discrepancies remain, and the robustness of the extrapolations needed for CR studies is still controversial. For more discussion see $[32,33]$.

\section{Air shower development}

The spectrum of cosmic rays extends over many orders of magnitude in energy. The flux falls very rapidly with energy and therefore a variety of techniques are used for observations in the different energy ranges. Instruments on balloons and satellites can directly detect the primary particles, but are limited to the low energy range where the CR flux is sufficiently large. At higher energy the fluxes of cosmic rays can only be studied with "indirect" methods, that is observing the showers that they generate in the atmosphere. In this case, as already discussed, one has the problem to determine the identity and estimate the energy of the primary particles from the data collected by the sensors that measure some properties of the showers. This requires to have a sufficiently good understanding of the development of CR showers in air, and therefore a sufficiently precise description of the properties of hadronic interactions.

A very brief outline of the development of a proton cosmic ray shower can be summarized as follows. The primary particle penetrates the atmosphere and interacts after crossing a column density $X_{0}$. The exponential distribution of $X_{0}$ is determined by the interaction length $\lambda_{p}\left(E_{0}\right) \propto \sigma_{\text {prod }}^{-1}$. Elastic and quasi-elastic (target nucleus fragmentation) processes can, to a good approximation, be neglected because they result only in a small energy loss for the primary particle, and one needs therefore to have a precise knowledge of the "production cross section" $\sigma_{\text {prod }}$ that corresponds to the inelastic processes where the new hadronic particles are produced.

From the interaction point emerge several (at high energy many) particles that carry the 4-momentum of the primary proton. It is crucial to have a good description of the structure and composition of the final state, and of the energy spectra of the secondary particles. After the rapid decay of hadronic resonances (such as $\rho, \omega^{\prime}$, $\Delta$ and so on) the final state is composed of quasistable hadrons that decay via the electromagnetic or weak interactions. In most cases one high energy "leading" baryon carries a large fraction of the primary energy, and a precise description of the energy spectrum of this particle is of particular importance for the shower development. Additional nucleons and anti-nucleons are present in the final state, some produced as fragments of the target nucleus, other generated by the creation of baryon/anti-baryon pairs in the interaction. The most abundant secondaries are pions of the three charges. Neutral pions decay rapidly into pairs of gamma rays $\left(\pi^{\circ} \rightarrow \gamma \gamma\right)$. These photons, together with additional ones generated by the decay of $\eta$ and $\eta^{\prime}$ mesons, are the source of an electromagnetic shower, that constitutes the largest component of the shower.

Charged pions can either interact or decay. The decay length of a particle grows linearly with momentum because of the Lorentz time dilatation $\left(\ell_{\mathrm{dec}}=c \tau p / m\right)$, while the interaction length decreases only logarithmically with energy, reflecting the cross section behaviour $\sigma_{\text {inel }} \propto \ln ^{2} s$. The result is that high (low) energy particles interact (decay). Note that the interactions of charged pions (and other mesons) in a shower also need to be precisely modeled, and at high energy this requires the extrapolation of results obtained in $p p$ and $\bar{p} p$ colliders. The decay of charged pions $\left(\pi^{+} \rightarrow \mu^{+} v_{\mu}\right)$ is the main source of the muon and neutrino components of the shower. Neutrinos are effectively invisible, while the observation of muons at 
ground level plays a crucial role in the identification of the primary particle.

$\mathrm{K}$-mesons (that contain a strange (anti)-quark) also account for a significant fraction (of order 10\%) of the final state energy in an interaction and their modeling is also very important for shower development. The production of particles that contain heavy ( $c$ and $b$ ) quarks is dynamically strongly suppressed, and in general is of negligible importance, however semileptonic decays can be an important source of background in the search for astrophysical neutrinos.

Showers generated by primary nuclei have essentially the same structure as for proton induced showers, but one has to consider some additional complications. In a nucleus-nucleus inelastic collision only a fraction of the projectile nucleons "participate" in the interactions, while the remaining "spectator" nucleons emerge from the interaction as an ensemble of fragments that travel with approximately the same velocity of the initial nucleus, and one needs an accurate method to calculate the distribution of the number of participating nucleons in a collision, and the properties of fragmentation for the spectators.

Because of its complex structure, the simplest and most accurate method for calculating the development of cosmic ray showers is the use of a Monte Carlo code. Such a code, beyond the description of hadronic interactions has to contain several other elements: the structure of the atmosphere; the electromagnetic processes that control the development of purely electromagnetic showers (bremsstrahlung and pair production) and the propagation of charged particles in matter (multiple scattering and ionization losses); the spectra of final particles in weak decays (with the relevant matrix elements). All these additional elements in a shower code are, however, under sufficiently good control, and the systematic uncertainties about the prediction of shower development have their origin in the description of the properties of hadronic interactions.

\section{Air Shower Observations}

Many air shower experiments only observe the particles that reach the ground level, effectively measuring a single layer of the shower development. The particles created in the shower, distributed with approximate cylindrical symmetry (broken by geomagnetic field effects and fluctuation effects) around the shower axis, are detected by arrays of detectors at ground level distributed with an appropriate density over a sufficiently large area. In most cases the observatory reconstructs the electromagnetic component of the shower ( $e^{ \pm}$and $\gamma$ 's) and the muon number at the detector level. If in addition the detector is capable of observing, close to the shower axis, the hadronic component of the shower, the quality of the measurement can be significantly improved. The distribution for the pair of observables $\left\{N_{e}, N_{\mu}\right\}$ can then be mapped into a distribution for the quantities $\left\{A, E_{0}\right\}$, that is the mass and energy of the primary particle (the number of photons does not give additional information, because the spectra of photons and $e^{ \pm}$are in "equilibrium" and to a good approximation one can predict one from the other). This program requires to have in hand the probability $p\left(N_{e}, N_{\mu} ; A, E_{0}, \theta\right)$ that a primary particle of mass $A$, energy $E_{0}$ and zenith angle $\theta$ generates a shower reconstructed with electron and muon numbers $N_{e}$ and $N_{\mu}$. Obviously, with increasing $E_{0}$, the electron and muon number increase, while a larger mass number $A$ (for a fixed primary energy) results in a larger muon number.

The program outlined above clearly depends on the model of hadronic interaction code used for estimating the response function $p\left(N_{e}, N_{\mu} ; A, E_{0}, \theta\right)$, and the spectra obtained by experiments like Tibet III, Kascade and Kascade-Grande are given with "labels", that indicate the Monte Carlo codes used for the shower simulation.

\section{The 'Fly's Eye" fluorescence light detection method}

The task of reconstructing the energy in a shower has been much simplified, and the systematic error in the energy determination has been much reduced after the introduction by the Fly's Eye experiment of the fluorescence light detection technique. The light emitted by the excitation of nitrogen molecules by charged particles in the shower can be detected to estimate the longitudinal profile of a shower, that is the number of charged particles $N(X)$ present at an atmospheric depth $X$. The energy that the shower dissipates in air as ionization can be calculated from the profile performing the integral:

$$
E_{\mathrm{ion}}=\langle|d E / d X|\rangle \int d X N(X) .
$$

The total energy of the primary particle is then obtained including corrections to take into account the missing energy transported by muons (that deposit their energy in the ground) and neutrinos:

$$
E_{0} \simeq E_{\text {ion }}+E_{\mu}+E_{\nu} .
$$

In this estimate, the model dependence enters only for the missing energy correction that is relatively small (approximately $20 \%$ for iron or $10 \%$ for protons at $10^{18} \mathrm{eV}$, and $12 \%$ for iron or $6 \%$ for protons at $10^{20} \mathrm{eV}$ ).

In hybrid experiments such as the Auger and Telescope Array observatories the fluorescence method is used to calibrate the measurement of a surface array, that can achieve larger exposures, since fluorescence light detection is only possible during dark (moonless) and clear (cloudless) nights.

The introduction of the fluorescence light method has allowed to obtain a reasonably precise measurement of the energy spectrum of UHECR that is independent from assumptions on the composition of the particles and the modeling of hadronic interactions, but one remains with the question of the composition of the particles.

As indicated in Eq. (4), the area under the curve of the longitudinal development $N\left(X ; E_{0}, A\right)$ of a primary of energy $E_{0}$ and mass $A$ is determined (to a good approximation) by the primary energy $E_{0}$, independently from $A$. On the other hand the shape of $N\left(X ; E_{0}, A\right)$ gives information about the mass of the primary particle, it is for example intuitive that the showers generated by a heavy nucleus are less penetrating than the showers generated by protons of the same total energy. The crucial problem is that the shape $N\left(X ; E_{0}, A\right)$ also depends on the properties 


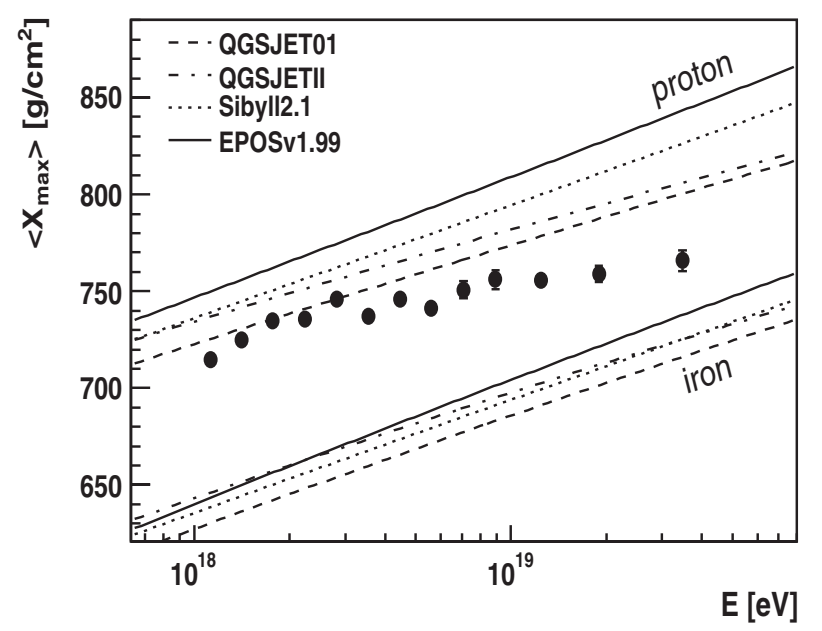

Figure 5. Average $X_{\max }$ measured by the Auger experiment [34] compared with air shower simulations using different hadronic interaction models.

of hadronic interactions. It is again intuitively clear that if the cross sections for hadronic interactions are larger (and the interaction lengths therefore shorter) or if the spectra of secondary particles generated in the interactions are softer, the showers of a primary of a given mass and energy develop faster. Programs to determine the composition of UHECRs from the shape of the longitudinal development are therefore critically dependent on the modeling of hadronic interactions.

The simplest way to characterize the shape $N(X)$ of a shower is to estimate the depth $X_{\max }$ where the shower size has its maximum. The average value $\left\langle X_{\max }\left(E_{0}\right)\right\rangle$ of all showers with energy $E_{0}$ depends on the composition of $\mathrm{CR}$ at that energy.

In Fig. 5 the points represent the data collected at the Auger observatory, [34,35], on the average $X_{\max }$ for showers of different energy, while the lines show predictions of $\left\langle X_{\max }(E)\right\rangle$ obtained using different Monte Carlo codes [27-30]. for showers generated by protons and iron nuclei.

Pierog [31] showed in his Fig. 12 that using the same set of codes tuned to LHC data there was a strong reduction in the modelling uncertainty in the predictions for $X_{\max }$ from $\sim 50 \mathrm{gm} / \mathrm{cm}^{2}$ to $20 \mathrm{gm} / \mathrm{cm}^{2}$.

The same experimental study has been performed by the HiRes and Telescope Array detectors [36,37]. Unfortunately the data of the different experiments are not in good agreement and a consistent interpretation of the data is not possible. This is very likely the consequence of not yet understood experimental systematic effects. One can however use the data (of any of the experiments) to discuss some important methodological points.

It should be ppinted out that in March 2015 (i.e. postSymposium) that a joint working group of Auger and the Telescope Array analyzed the (in)consistency of their determination of the primary composition and concluded that the data are consistent. The compatibility of the PA and TA results are addressed in more detail in [38].

The first important point is that for any model the average value of $X_{\max }$ for a constant mass $A$ grows approximately logarithmically with energy:

$$
\left\langle X_{\max }\left(E_{0}, A\right)\right\rangle \simeq X_{A}+D \ln E_{0}
$$

with an "elongation rate" $D$ that varies only slowly with energy. Since the average $X_{\max }$ of the showers of a primary of energy $E$ and mass $A$ is approximately equal to the $\left\langle X_{\max }\right\rangle$ of a proton shower of energy $E / A$ one also has:

$$
\left\langle X_{\max }\left(E_{0}, A\right)\right\rangle \simeq X_{\max }\left(E_{0}, p\right)-D \ln A
$$

In other words, having a robust prediction for the function $\left\langle X_{\max }\left(E_{0}, p\right)\right\rangle$, that in a first approximation, for a limited range of energy is determined by the two parameters (that determine a straight line in the plane $\left.\left\{\ln E, X_{\max }\right\}\right)$, one can map the experimentally observable $\left\langle X_{\max }\right\rangle$ into an estimate for $\langle\ln A\rangle$.

The program outlined above is of course limited by uncertainties in the modeling of the hadronic interactions that determine the parameters $X_{p}\left(E_{0}\right)$ and $D$. From Fig. 5, one would conclude that the average mass of the cosmic rays becomes heavier with increasing energy, but for example the composition at $E_{0} \simeq 2 \times 10^{18} \mathrm{eV}$ is pure protons for the QGSJET01 model but contains a significant amount of nuclei using the EPOS model.

It is interesting to note that for a purely electromagnetic shower (generated by an $e^{ \pm}$or photon) the position of the shower maximum grows logarithmically with the primary particle energy, with a slope equal to the radiation length in air, $\lambda_{0}$ :

$$
\left\langle X_{\max }\left(E_{0} ; \gamma\right)\right\rangle=\lambda_{0} \ln \left(E_{0} / \varepsilon\right)
$$

(where $\varepsilon$ is the critical electron energy in air). An analytic derivation of this result is given for example in Rossi and Greisen [39]. The logarithmic dependence on the energy can be understood qualitatively as the consequence of the fact that an energy independent radiation length determines the interaction points for the two processes (bremsstrahlung and pair production) that generate the shower, and in both cases the energy of a particle is "split" in an energy-scale independent way. These arguments are illustrated pedagogically in the well known toy model introduced by Heitler [40] where the shower is formed by a single (electron/photon) particle type that after a "splitting length" $\lambda$ divides into two particles each with one half the energy, until the critical energy $\varepsilon$ is reached. The shower develops to the maximum size $N_{\max }=E_{0} / \varepsilon$ at the depth $X_{\max }=\lambda \log _{2}(E / \varepsilon)$.

In the case of hadronic showers one must include the effects that the interaction lengths are decreasing with energy and that with the growth of the interacting particle energy particles in the final state increase in multiplicity and become progressively softer. The main difference between the models shown in Fig. 5 is associated to progressively stronger softening of the inclusive spectra going from EPOS (the most penetrating showers) to QGSJET01 (least penetrating showers).

Some natural and important questions emerge immediately. Which model best represents our current understanding of hadronic interaction? Do the differences 
between the models give a reasonable estimate of the theoretical uncertainties? The spread of the predictions of the available models is often taken as an estimate of the systematic error associated to the theoretical uncertainties on hadronic interactions (using implicitely the assumptions that all models are of reasonably good quality). It is however also, at least logically, possible, that all models are incorrect, and that some new unexpected phenomena become important at high energy. One should also remember that all the models in use for CR simulations attempt to describe the same experimental results, and share a number of common assumptions (or perhaps prejudices). If the results have systematic errors, or the assumptions are flawed, this could lead to incorrect (and similar) predictions for all models.

The methodology outlined above (where one first constructs a model for hadronic interactions and then uses the model to determines the CR composition) could perhaps also be reversed. There are in fact measurements that give information about the composition of cosmic rays, and are completely independent from the modeling of hadronic interactions. One such method uses the Milky Way as a magnetic spectrometer, measuring the deviation of particles generated by known sources. Having a sufficiently precise knowledge of the galactic field, one can calculate the electric charge of the particles (the momentum is measured experimentally). A second possibility is to interpret features in the CR spectrum as the imprints of energy losses on a smooth injection spectrum. A robust identification of the origin of some spectral features (for example the GZK suppression for pion photoproduction by protons, or the "dip" associated to the threshold for $e^{+} e^{-}$pair production ) could allow to estimate the composition of the detected particle. It would then be possible to use the observed properties of shower development to obtain information about hadronic interactions.

As an illustration, assuming that most of the UHECR particles are protons (as advocated in some astrophysical scenarios), the current Auger data on $\left\langle X_{\max }(E)\right\rangle$ imply that the showers are much less penetrating than the current predictions. This in turn would imply that the inelastic cross sections are larger, and/or that the inclusive spectra in the final state are softer than expected.

More in general one can hope (but in fact also realistically expect) that, in future, it will be possible to simultaneously determine the composition of UHECR and the relevant properties of hadronic interactions. The key here is the consistency of different measurements of shower development. One very interesting example of this possibility is the recent measurement of the proton interaction length in air obtained by the Auger collaboration [7] for the observations of CR with energy $E_{0} \simeq 10^{18.24} \mathrm{eV}$ ( $\sqrt{s} \simeq 57 \mathrm{TeV}$ for nucleon-nucleon interactions). The result: $\sigma=505 \pm 23$ (stat) ${ }_{-36}^{+28}$ (sys) $\mathrm{mb}$ for the "production" cross section, on an average air nucleus is obtained with only very weak assumptions about the amount of protons in the CR spectrum at the energy considered. The result can be translated in an estimate for $\sigma_{\text {tot }}^{p p}$ as shown in Fig. 1.
For more discussion on the analysis of the composition of the UHECR see [41-43].

\section{Astrophysics of cosmic rays}

\section{The acceleration and propagation problems}

The population of cosmic rays is formed by particles that have been accelerated by many different sources that were active, probably for only an (astrophysically) short time interval, at different epochs in the past and at different points in space. The identification of the astrophysical accelerators, and the understanding of their structure, and of the mechanisms that accelerate charged particles to relativistic energy can be seen as the main scientific goal of high energy astrophysics.

It is now clearly established that most of the CR particle that we observe near the Earth are of galactic origin, and have been produced by sources inside our Galaxy. Gamma ray observations, measuring the emission along different lines of sight can trace the density of cosmic rays in different points in space, or more precisely the product of the densities of CR's and gas. The observations show that cosmic rays form a halo around the visible disk of the Milky Way, where the CR density is much larger than present in the near extragalactic space. This CR halo is formed by the galactic magnetic field that can confine, for a finite time, the charged particles. The contribution of extragalactic sources to the CR flux is expected to become dominant only at very high energy. The determination of the critical energy $E^{*}$ where the galactic and extragalactic components are equal is one of the key open problems in the field. A natural possibility is that the energy $E^{*}$ coincides with so called "ankle" at $E \simeq 10^{19} \mathrm{eV}$, but a transition at lower energy is also possible.

The shape of the energy spectra of the galactic cosmic rays is therefore shaped by two effects: acceleration and propagation. Charged particles of type $j$ are first accelerated in their sources and released in the interstellar medium with spectrum $Q_{j}(E)$, and then remain partially confined in the Galaxy for a finite time. The shape of the observable spectrum $\phi_{j}(E)$ is not identical to the interstellar injection spectrum, because it can be distorted by propagation effects.

The simplest model to illustrate this point is the so called "Leaky Box" model, where the time evolution of the number of cosmic rays of type $j$ and energy $E$ in the Galaxy is described by the equation:

$$
\dot{N}_{j}(E)=Q_{j}(E)-\frac{N_{j}(E)}{T(E)}-\sigma_{j}(E) \beta c\left\langle n_{\text {ism }}\right\rangle N_{j}(E) .
$$

The first term on the right side of the equation is the (space integrated) injection, the second term accounts for escape from the Galaxy with a characteristic time $T$ that is expected to be a function of the particle rigidity (and therefore common to different particle species), and the last term describes the absorption for interaction with interstellar gas. In Eq. (9) energy losses and reacceleration are neglected. For a stationary condition 
$\left(\dot{N}_{j}=0\right)$ the solution of the equation is:

$$
N_{j}(E)=Q_{j}(E)\left[\frac{1}{T(E)}+\sigma_{j}(E)\left\langle n_{\mathrm{ism}}\right\rangle \beta c\right]^{-1} .
$$

The observable energy distribution $N_{j}(E)$ is now given as the product of the injection spectrum and a residence time $T(E)$ that accounts for propagation effects, with a correction for interactions. The problem is to understand the role of the different effects.

It is of course possible to disentangle the effects of injection and propagation if one of the two terms is known. This is the case for the injection of secondary nuclei, such as Lithium, Beryllium and Boron that are very rare in nature, but are relatively abundant in cosmic rays. Secondary nuclei in the cosmic rays are not directly accelerated but are produced as relativistic particles by the fragmentation of higher mass nuclei. For example the injection of Boron, in the approximation where Carbon is the dominant source can be estimated as:

$$
Q_{B}(E)=N_{C}(E) \sigma_{C \rightarrow B}(E) \beta c\left\langle n_{\text {ism }}\right\rangle
$$

where $E$ is the energy per nucleon, that remains approximately constant in nuclear fragmentation. Combining Eqs. (10) and (11) one finds:

$$
T(E)\left\langle n_{\mathrm{ism}}\right\rangle \beta c=\frac{R(E)}{\sigma_{C \rightarrow B}(E)-R(E) \sigma_{B}(E)},
$$

where $R(E)=N_{B} / N_{C}$ is the ratio between the fluxes of Boron and Carbon. The crucial point is that the CR residence time, or more precisely the average column density crossed by cosmic rays while confined in the Galaxy can be inferred from measurements of the ratio of the fluxes of secondary and primary nuclei, if the relevant cross sections $\sigma_{j}(E)$ and $\sigma_{j \rightarrow k}(E)$ (absorption and fragmentation) are sufficiently well known.

The discussion we have outlined above has been performed in the Leaky Box model, that neglects the space dependence of the CR injection and density, and is therefore manifestly inadequate for a realistic study. This is not the place for a more complete discussion, the simplest model should be sufficient to illustrate the point that a knowledge of the energy dependence of the absorption and fragmentation cross sections for different nuclei is essential for the determination of the properties of propagation of cosmic rays in the Galaxy. Very valuable information on these cross sections has been obtained, but additional measurements in a more extended energy range are very desirable.

\section{Structures in the spectra}

Until recently, it was common wisdom to assume that the spectra of protons and nuclei are smooth, and reasonably well approximated with simple power laws from a lower energy of few $\mathrm{GeV} /$ nucleon (when mass and solar modulation effects become negligible) up to the "knee energy" (of order $E_{\text {knee }} \simeq 3 \times 10^{15} \mathrm{eV}$ ). This common wisdom has been put in question by measurements of the balloon-borne experiment CREAM that has made several long duration flights in Antarctica. The analysis of the first 70 days of cumulative data taking [44] has indicated that the spectra of protons, helium and other higher $Z$ nuclear species in the energy range accessible to the experiment (few $\mathrm{TeV}$ to $\sim 100 \mathrm{TeV}$ for protons) is harder than the extrapolation from lower energy measurements. The CREAM collaboration has called this effect the "discrepant hardening" of cosmic rays, observing that to join the extrapolations of the low and high energy bands one needs to introduce a hardening of the energy spectrum at a rigidity of order $200 \mathrm{GV}$.

This hypothesis has received a spectacular confirmation from Pamela that has directly measured the proton and helium spectra in the rigidity range $1 \mathrm{GV}$ to $1.2 \mathrm{TV}$ [45] and found that the spectral shapes are not compatible with a simple power law and exhibit a sharp ankle-like feature at a rigidity of approximately $220-230 \mathrm{GV}$. The results challenge the simple picture of $\mathrm{CR}$ acceleration and propagation that has been commonly accepted for a long time, and a confirmation of the existence of this structure in the CR spectra would certainly have important consequences. The effect could be associated to either the acceleration or the transport of cosmic rays, and a variety of possible explanations is under discussion. The result has been recently confirmed for the proton spectrum by the AMS measurements of the proton flux [46].

\section{Anisotropies}

The flux of the Galactic cosmic rays is nearly isotropic because of the effect of the Milky Way magnetic fields. However the isotropy is not perfect, and small, energy dependent anisotropies remain and encode very valuable information about the cosmic ray properties, the distribution of their sources, the Galactic magnetic fields and our heliospheric environment.

The anisotropies are however difficult to measure. The study of these effects has recently received new attention, and there are several new, interesting results. The Tibet Air Shower Array published a "quasi" two-dimensional high precision anisotropy measurement for energies between a few $\mathrm{TeV}$ to a few hundred $\mathrm{TeV}$ in 2006 [47]. The "quasi" means that the map is really an ensemble of one-dimensional maps in right ascension with a fixed declination $\delta$. This is because points on the celestial sphere with the same declination follow the same trajectory in the sky above the detector and therefore the relative intensity of the fluxes from points with the same declination can be compared directly without the need of a precise modeling of the zenith angle dependence of the detector response. The "quasi" two-dimensional results of Tibet show nearly dipolar features with an amplitude of order $0.1 \%$ that decreases gradually when the energy grows from 4 to $300 \mathrm{TeV}$.

The Tibet results have been repeated and extended (at lower energies) by the observations of Milagro [48], ARGO [49] and more recently IceCube [50]. The amplitude of the anisotropy measured by ARGO grows with energy between 0.7 and $3.9 \mathrm{TeV}$. There is not yet an accepted explanation for the non trivial energy dependence of the observed large scale anisotropy.

The Milagro observations have also shown [51] the existence of two "localized regions of excess of unknown 
origin" with dimensions of order 10 degrees, that have become known as the "Milagro hot spots". The excess is attributed to hadronic showers (not photons) with an energy spectrum harder than the average one. The existence of these surprising localized excesses has been confirmed by ARGO [49] observing the same region of the sky. The results of IceCube [50] (obtained using downgoing muons and therefore observing the southern celestial hemisphere) confirm the existence of intermediate angular scale excesses in the sky.

Shortly after the announcement of the Milagro result, Drury and Aharonian [52] discussed the difficulty of finding a consistent explanation for the "puzzling Milagro hot spots". A few ideas (such as the existence of "magnetic mirrors", or of source rich regions) have been proposed as solutions, but the problem remains open.

To conclude this short discussion about anisotropies it should be added that it remains very puzzling that one has not detected larger anisotropy effects at higher energy (at the knee and above). One expects that at a sufficiently high rigidity the confinement of cosmic rays by the Milky Way magnetic field should begin to fail, and then one should observe the development of a large anisotropy. The $\mathrm{CR}$ angular distribution should return to isotropy when extragalactic cosmic rays become dominant. Finally, at the highest energy, when the cosmic ray horizon becomes restricted by propagation effects to the "local universe", one should again observe anisotropies, now associated to the structures created by gravity on the scale $10-100 \mathrm{Mpc}$. Perhaps a hint of the last effect is present in the Auger data, but the effects on the CR angular distribution of a transition from a galactic to an extragalactic cosmic ray population have not been observed.

\section{The "Knee"}

The interpretation of the so called "knee", a sharp steepening of the cosmic ray spectrum at an energy $E_{\text {knee }} \simeq 3 \times 10^{15} \mathrm{eV}$ remains controversial. The analysis of the data of the Kascade detector [53], performed measuring simultaneously the electron and muon numbers of the showers, determines the energy spectrum and estimates the evolution of the chemical composition of the primary cosmic rays. The Kascade results suggest that the "Knee" is associated to a steepening of the light (proton or helium) components of CR flux. This conclusion, however, depends crucially on the modeling of the cosmic ray showers, and has therefore a systematic error that is difficult to estimate with reliability.

In a large set of models, the cosmic ray spectrum is rigidity dependent. This means that if the $p$ spectrum presents a feature at energy $E_{p}$, a nucleus of charge $Z$ should show the same feature at energy $E_{p} \times Z$. If the observed knee is produced by the steepening of protons, the spectrum at higher energy should show a sequence of steepenings of different nuclear species with increasing $Z$, with perhaps the most easily observable effect the steepening of iron at energy $26 \times E_{\text {knee }}$.

The Kascade-Grande collaboration has reported [54] the observation of a steepening in the cosmic ray energy spectrum at about $8 \times 10^{16} \mathrm{eV}$. The structure is seen in the all-particle energy spectrum, but it becomes much more significant selecting showers associated with heavy primaries, so that the result appears to support a view of the knee as a rigidity dependent structure.

\section{Ultra High Energy Cosmic Rays}

The study of the highest energy particles, that are almost certainly of extragalactic origin, continues to be at the center of discussions, speculations and controversy. For many years the dominant question in this field has been the possible existence of a large flux of particles with energy larger that $10^{20} \mathrm{eV}$, above the energy where intergalactic space becomes non-transparent to protons (because of photoproduction interactions on the CMBR) and nuclei (because of photodisintegration processes). These results generated many interesting speculations, like the existence of an additional source of particles of extremely high energy from the decay of super-heavy (mass of order of the GUT mass, $M_{\mathrm{GUT}} \simeq 10^{25} \mathrm{eV}$ ) particles, or the existence of violations of Lorentz invariance, that suppress the existence of reaction thresholds.

The results of the HiRes [55], and Auger [56] observatories have clearly demonstrated the existence of a sharp steepening of the CR flux at an energy $E_{\mathrm{UHE}} \simeq$ $(4-6) \cdot 10^{19} \mathrm{eV}$. With this result the phenomenological motivations for "exotic physics" in UHECR have essentially disappeared. One remains with a fascinating and difficult astrophysical problem: where and how are particles accelerated up to these quite extraordinary energies?

The nature of the steepening in the energy spectrum of the UHECR has not yet been determined, and one has three possible explanations for the effect. The first one is that we are simply observing the "end point of the accelerators", and $E_{\mathrm{UHE}}$ is the maximum energy that can be obtained in the sources. On the other hand it is also possible that the steepening is a propagation effect, produced by the interaction of $\mathrm{CR}$ particles with the photons of the CMBR. In the case of protons the energy loss becomes important above the threshold for pion production $E_{p} \gtrsim\left(m_{p} m_{\pi}\right) / \varepsilon$ (with $\varepsilon$ the typical energy of the CMBR photons), an effect predicted by Greisen, Zatsepin and Kuzmin. For nuclei the important reaction is photodisintegration, with energy threshold $E_{A} \gtrsim m_{A} \epsilon_{b} / \varepsilon$ (with $\epsilon_{b}$ the binding energy on a single nucleon in the nucleus). The photodisintegration energy threshold grows approximately linearly with $A$ (since $\epsilon_{b} \simeq 8 \mathrm{MeV}$ is nearly independent of $A$ ), and therefore iron is the nucleus with the highest threshold. The bottom line is that the particles at the highest energies (close to and above the UHE steepening) are very likely only protons and/or iron. Discriminating between a proton or iron CR composition from the shape of the UHE steepening is in principle possible, but in practice difficult, because the spectral distortions generated by energy losses on protons and iron are similar. The discrimination also requires good control of the absolute scale of energy determination.

The HiRes group has in fact claimed [55] that their measurement of the energy spectrum is well fitted with a proton dominated composition, and that they have observed the Greisen-Zatsepin-Kuzmin suppression. The results of the Auger collaboration are not incompatible 
with this conclusion (allowing for a rescaling of all measured energies), but this important problem should be considered as still open.

\section{Galactic to Extragalactic transition}

The highest energy cosmic rays are nearly certainly of extragalactic origin. The very simple argument is related to the confinement of charged particles by magnetic fields. The Larmor radius of a proton (iron nucleus) with energy $10^{20} \mathrm{eV}$ in a field $B \simeq 3 \mu$ Gauss (the typical value in the disk of the Milky Way) is $r_{L} \simeq 36 \mathrm{Kpc}(1.4 \mathrm{Kpc})$. Considering the visible dimension of our Galaxy (a disk radius of order $15-20 \mathrm{Kpc}$ ), it appears very unlikely that the magnetic halo of the Galaxy can be sufficiently extended to confine and isotropize the highest energy particles, even if they are iron nuclei.

This implies that there is a transition energy $E^{*}$ where the Galactic and extragalactic contributions to the $\mathrm{CR}$ spectrum are equal, with the galactic (extragalactic) component dominating below (above) $E^{*}$. The identification of this transition energy is emerging as a key problem for cosmic ray studies.

The "Ankle" (a flattening in the energy spectrum at $E_{\text {ankle }} \simeq 5 \cdot 10^{18} \mathrm{eV}$ ) has been for a long time interpreted as the galactic to extragalactic transition energy. A few years ago, Berezinsky and collaborators [57] have suggested a very different interpretation of the flattening. These authors assumed that all cosmic rays with energy $E \gtrsim 10^{18} \mathrm{eV}$ (below and above the "ankle") are extragalactic protons and that they are generated at their sources with a smooth, featureless power law spectrum. The effects of propagation can then generate the observed spectral features. The "ankle" is really a "dip", produced by the opening of pair production in $p \gamma$ interactions as a new channel for energy loss above a threshold $E \simeq$ $\left.m_{p}\left(2 m_{e}\right) / \varepsilon\right)$. The name "dip model" encodes this new dynamical explanation and renames the structure in the spectrum.

In the dip model the galactic to extragalactic transition energy is therefore at lower energy, and it has been suggested that is associated with a steepening of the spectrum known as the "second knee" (for a discussion for the observational evidence for this not very prominent structure see [58]). The fact that the transition from a softer galactic component to a harder extragalactic component is marked by a steepening is surprising and needs some fine tuning (see discussion in [59]). The dip model is very predictive, because it predicts the deviations of the spectrum from a simple power law, and a pure proton composition.

Changing the transition energy has very important consequences. In the models with the transition at the Ankle it is necessary to assume that our Galaxy contains sources capable to accelerate particles up to $10^{19} \mathrm{eV}$ and above. In the dip model one has a less stringent (by a factor 10 or more) requirement for the maximum energy of the Milky Way accelerators, but on the other hand the power density requirement for the extragalactic sources is dramatically increased. The identification of the transition energy $E^{*}$ can therefore be seen as a central problem with wide and important implications. The identification of $E^{*}$ can, in principle, be performed combining measurements of the spectral shape, the composition and the anisotropy of the CR flux.

\section{Cosmic Ray Point Sources}

At a sufficiently high energy, the deviations of charged particles propagating across the astrophysical magnetic fields could become sufficiently small so that their directions of arrival point to their sources. This would allow to perform astronomy with cosmic rays. If this expectation is confirmed by the data, the consequences would be dramatic, as the study of the high energy sources would greatly benefit by the possibility of "multimessenger" observations.

The concept of CR astronomy received an impressive boost when in November 2007 the AUGER collaboration published a result [35] that indicated that the highest energy events $\left(E \geq 6 \cdot 10^{19} \mathrm{eV}\right)$ are correlated with the position of a nearby $\mathrm{AGN}\left(z \leq 0.017\right.$ or $\left.d_{\min }=75 \mathrm{Mpc}\right)$, with a correlation angle of $3.1^{\circ}$. The Auger study has been updated in September 2010 [60] with results that significantly dilute the statistical significance of the effect, without, however, erasing it entirely.

The existence of the correlation has not been confirmed in the observations of the Yakutsk [61] or HiRes [62] detectors. The result is potentially very important, and has generated a large amount of discussion and controversy. Taken at face value the existence of the correlation suggest several important conclusions. The first one is that the highest energy particles have a small electric charge, and are protons or perhaps helium nuclei. The total deviation of a particle can be considered as the combination of contributions accumulated in the source envelope, intergalactic space, and inside our Galaxy.

$$
\delta \theta \simeq \delta \theta_{\text {source }} \oplus \delta \theta_{\text {extra }} \oplus \delta \theta_{\mathrm{MW}} .
$$

The "Milky Way" contribution can be written (assuming the deviation is small) as:

$$
\delta \theta_{\mathrm{MW}} \simeq \frac{q_{e} Z}{E}\left[\int d \ell B_{\perp}^{\mathrm{MW}}(\ell, \Omega)\right]
$$

From our knowledge of the magnetic fields of our Galaxy one can estimate (or put limits) on the integral of the transverse field along different lines of sight. From the upper limit $\delta \theta_{M W} \lesssim 3.1^{\circ}$ one obtains an upper limit on the electric charge $Z$ that is of order $Z \lesssim 2$. In addition, the small correlation angle implies a significant limit on the intensity and correlation length of the extragalactic magnetic fields.

The result does not imply that AGNs are in fact the source of the highest energy particles because (as clearly discussed in the Auger paper), the AGNs themselves are spatially correlated with other normal galaxies, and more in general with the distribution of (ordinary) matter, so alternative theories for the site of the acceleration of UHECR remain viable.

It is however remarkable that the "hottest" region of the Auger sky (above $6 \cdot 10^{19} \mathrm{eV}$ ) is around CenA. This is the AGN closest to us, in fact very near at a distance of only 3-4 Mpc in the direction of Centaurus, that has 
been considered as an important source of UHE particles for a long time. A cone of 3 (18) degrees around the CEN A position contained 3 (8) events. Is this statistically significant? And what does it mean? The answer to the first question is likely: yes, even if the statistical significance remain modest. The possible consequences of the identification of CenA as a source of UHE are the subject of a very interesting discussion. A problem for the interpretation is that it is possible that the UHE particles are emerging from the vicinity of the central super massive black hole or at large distances from it, from the large radio lobes produced by the interaction of the AGN jets with the intergalactic medium.

It is immediately clear that there is significant tension (if not an explicit internal contradiction) between the results on the shower longitudinal profile, and those of the magnetic deviation. The first ones indicate that around $E \simeq 3 \cdot 10^{19} \mathrm{eV}$ the composition is dominated by heavy nuclei, the second suggest that at energies only a factor of 2 higher the composition is dominated by protons (or perhaps helium). Is it possible that one is observing a very rapid composition change? Perhaps the results on the correlations with AGN are a statistical mirage? Jim Cronin has remarked [63] "... these results also demand a more careful review of what seemed to be an obvious conclusion that iron nuclei could not show an anisotropy because of galactic and perhaps extragalactic magnetic felds.". All of this remains the subject of an intense discussion.

\section{Electrons, positrons, anti-protons and Dark Matter}

The study of the spectra of sub-dominant particles in cosmic rays: $e^{-}, e^{+}$and $\bar{p}$ is certainly the topic that has received most attention in recent years. The intensity of the interest is a consequence of the fact that positrons and anti-protons are crucial probes to study the existence of Dark Matter (DM) in the form of WIMPs.

The study of the electron spectrum has the potential to give very important information about the properties of CR acceleration and propagation in the Galaxy. For protons and nuclei the energy loss during propagation can be safely neglected. However, on the contrary for $e^{\mp}$ energy losses via synchrotron radiation and Compton scattering (with the dependence $-d E / d t \propto E^{2}$ ) can be very important and determine the shape of the observed energy spectrum. The simultaneous study of the shape of the spectra of electrons and protons allows therefore to obtain information about the injection spectra of the two particle types and about the structure and properties of the Milky Way magnetic fields. The spectra of electrons and positrons depend also on the spatial distribution of the sources, because the observed $e^{\mp}$ can only arrive from a volume that rapidly decreases with energy.

It has been commonly accepted that anti-protons and positrons are not directly accelerated but are produced as secondaries by the interactions of CR with the interstellar medium in reactions such as $p+p_{\text {ism }} \rightarrow p p p \bar{p}+$ pions. The main source of $e^{+}$is the chain decay of positive pions produced in the same interactions. Electrons should be coaccelerated with protons and injected with a spectrum of similar shape (but lower maximum energy). The existence of DM in the form of WIMPs would add an extra-source of particles as product of the self-annihilation of the DM particles.

The recent measurement of the $\bar{p}$ by Pamela [64] is consistent with the standard picture, but the measurement of positrons by Pamela [65] indicates that the spectrum is much harder than expected. Observations of the electron flux by Pamela [66] and of the sum of electrons and positrons by Fermi [67] indicate that also the energy spectrum of electrons contains a hard component that emerges at high energy.

In a nutshell: the data indicate that the observed positron energy spectrum is as hard or harder than the proton spectrum in the range $E \sim 10-100 \mathrm{GeV}$, and the $e^{-}$flux also contains such a hard component. This is incompatible with the hypothesis that positrons are secondary particles, and also that electrons are coaccelerated with protons and injected with the same spectrum, and requires the existence of sources of $e^{ \pm}$that inject particles with a very hard spectrum (for a power law injection the required slope is of order $\alpha=1.7$ ). The annihilation of a DM particle of sufficiently high mass could provide an $e^{ \pm}$injection of the required shape, but the solution has the important difficulties discussed in the previous section.

There are alternative explanations for the excess of positrons that introduce new astrophysical sources of positrons, that at this point should probably be considered as more plausible. Pulsars have been proposed $[68,69]$ as the source of hard (and equal) spectra of $e^{ \pm}$generated by the acceleration of seed particles formed by pair creation in the magnetosphere of the objects. Considering the cumulative power output of young pulsars in our Galaxy, this mechanism could provide the required luminosity. However, the details of the acceleration and release of the particles remain very speculative.

Other ideas have also been put forward, and the problem of the acceleration of electrons and positrons (in the same sites where protons and nuclei are accelerated, or in different classes of sources) remains at the center of great theoretical and observational attention. For reviews of the status of possible interpretations of the positron result see [70].

\section{Dark Matter and Cosmic Rays}

The "Dark Matter problem" is recognized as perhaps the most important open problem in physics today, it can be seen as an "anomaly", a discrepancy between experimental observations and theoretical predictions. It is expected that a solution of the problem will require "new physics" that is an extension or modification of our currently accepted theoretical framework.

There is very strong evidence that the universe is filled with a form of matter that does not emit or absorb electromagnetic radiation, and whose existence is only deduced from the observations of its gravitational effects. From cosmological studies [71] one infers that the average density of the dark matter is approximately $26.1 \%$ of the critical density $\rho_{c}=3 H_{0}^{2} /(8 \pi G)$, while ordinary "baryonic" matter (composed of nucleons and electrons) 
accounts for only $4.8 \%$ of the critical density. The evidence of the dark matter exists also at smaller scales, in clusters of galaxies and in individual galaxies.

Of crucial importance for studies on the nature of the Dark Matter, is the fact that also the mass of our own Milky Way appears to be dominated by an invisible component. The mass distributions of a galaxy can be studied measuring (from Doppler shift) the rotation velocity of gas and stars as a function of distance from the galactic center. Since the early 1970s these studies show that for large $r$, beyond the extension of luminous matter, the radial velocity becomes approximately constant in contradiction with the Newtonian dynamics prediction of a dependence $\propto 1 / \sqrt{r}$, indicating that galaxies are embedded in a much larger DM halo.

Observations in our Galaxy give an estimate of the DM density in the vicinity of the Sun (and therefore also in an Earth laboratory) of order $\rho_{\chi} \simeq 0.3 \mathrm{GeV} / \mathrm{cm}^{3}$ (using $\chi$ as the label for the DM particle). Assuming a stationary condition, the space dependence of the mass density determines the shape of the velocity distribution of the DM particles, that have trajectories entirely determined by gravitation.

Since the evidence for dark matter is deduced from observations of the dynamics of visible matter under gravitational effects, it seems logically possible to describe the dynamics of visible matter without requiring the existence of an invisible source of gravity, but introducing some modifications to the laws of gravitation. This line of research, that is the attempt to construct MOdified Newtonian Dynamics (MOND) theories [72,73] capable of fitting the observations is an active subject of research, but it is disfavored. If, on the other hand, one assumes that general relativity is valid, and that an invisible form of matter is present and causes the anomalies observed in different astrophysical and cosmological systems, then the question of the nature of this invisible matter becomes pressing. It is remarkable that the study of the properties of the DM, leads to the conclusion that it cannot be formed by any of the known fields that are part of the Standard Model of particle physics. The existence of the DM requires a "paradigm shift", some form of "new physics".

Several different ideas have been put forward about the nature of the DM. Perhaps the most attractive (and probably also the simplest) idea, is the introduction of a new stable particle, sufficiently coupled with ordinary matter, so that is has been in thermal equilibrium in the early universe. Some of these particles will survive at the present epoch as a "thermal relics". Under very general conditions, the mass density of a thermal relic particle (indicated as $\chi$ ) is independent of its mass and is inversely proportional to the (velocity averaged) self-annihilation cross section $\langle\sigma v\rangle$ :

$$
\Omega_{\chi} \simeq \frac{3 \times 10^{-26} \mathrm{~cm}^{3} \mathrm{~s}^{-1}}{\langle\sigma v\rangle}
$$

(with the velocity average is performed at the 'freeze out' time when the distribution of the particle $\chi$ goes out of thermal equilibrium). For $\Omega_{\chi} \simeq 0.2-0.3 \mathrm{Eq}$. (15) implies an annihilation cross section with the order of magnitude characteristic of the weak interaction. In fact, writing $\sigma \sim \alpha^{2} / M^{2}$, with $M$ the mass of an intermediate particle that mediates the annihilation, one obtains $M \sim 200 \mathrm{GeV}$, the mass scale of the weak interaction. In this scenario the particles that constitute the DM are called WIMP's (Weakly Interacting Massive Particles).

Theoretical considerations, motivated by the desire to construct extensions of the Standard Model of particle physics that address some of its limitations, and are completely independent of the astronomical and cosmological observations discussed above, have lead to the prediction of the existence of new, yet undiscovered particles with mass close the Weak scale, and that can automatically provide the DM in the form of WIMP's. The most intensely discussed extension of the Standard Model that predicts the existence of DM is the introduction of Supersymmetry, that associates to each bosonic field a fermionic one with the same mass and quantum numbers (and viceversa). It is also predicted that the supersymmetric particle with the lowest mass should be stable, and would therefore be a natural candidate for the dark matter.

The WIMP concept has the attractive feature that it can be tested experimentally in a large fraction of its parameter space. There are in fact three "roads" to the discovery of WIMP's.

The first road, is the direct production in high energy accelerators in particular at the LHC. The supersymmetric particles should be produced in pairs, and each of the particles should quickly chain-decay into the stable state (the DM particle) that traverse the detector unobserved, leaving signature of missing energy and momentum. The search for supersymmetric particles is one of the main goals of the LHC at CERN. The experimental studies have until now only resulted in upper limits [74] and future data will provide more stringent test to the hypothesis of Supersymmetry.

The second road for the discovery of DM in the form of WIMPs is the observation of the elastic scattering of the DM particles with ordinary matter. DM particles in an Earth laboratory have an average velocity of the same order of the Sun rotation velocity around the center of the Galaxy $\left(v_{\odot} \simeq 200 \mathrm{~km} / \mathrm{s}\right)$. These particles are therefore non-relativistic and each one carries a kinetic energy of order of tens of $\mathrm{keV}$.

$$
\left\langle E_{\chi}^{\mathrm{kin}}\right\rangle \simeq\left[\frac{m_{\chi}}{100 \mathrm{GeV}}\right]\left[\frac{\langle v\rangle}{300 \mathrm{~km} / \mathrm{s}}\right]^{2} \mathrm{keV} .
$$

A nucleus (at rest in the laboratory frame) will be bathed by a large flux of DM particles $\left(\phi_{\chi} \sim\left(\rho_{\chi} / m_{\chi}\right)\left\langle v_{\chi}\right\rangle\right)$, and will absorb kinetic energy with a rate determined by the elastic scattering cross section. Integrating over the velocity of the DM particle and the scattering angle of the collision, it is straightforward to compute the recoil energy spectrum for each nucleus. Observations of this effect is the challenging goal pursued by several experimental groups using different detection methods and different target nuclei.

The third road to explore the WIMP hypothesis are the so called "indirect searches". If dark matter is composed of WIMPs, it is in fact not perfectly "dark", because it has a self-annihilation cross section that is approximately 
known from Eq. (15). The self-annihilation of the DM particles generates (after chain decay of unstable particles) an output of particles such as photons, electrons, protons, neutrinos (and their antiparticles). The production of these particles in the Milky Way is in principle observable. If $\rho_{\chi}(\vec{x})$ is the dark matter density at the point $\vec{x}$, the power density emitted from DM annihilation processes from the same point is (assuming Majorana particles):

$$
\frac{d L_{\mathrm{DM}}}{d^{3} x}(\vec{x})=\frac{\rho_{\chi}^{2}(\vec{x})}{m_{\chi}}\langle\sigma v\rangle .
$$

Integrating over the entire volume of the Galaxy one obtains a total luminosity of order:

$$
L_{\mathrm{DM}} \simeq 3 \cdot 10^{37}\left[\frac{\langle\sigma v\rangle}{3 \cdot 10^{-26} \mathrm{~cm}^{3} / \mathrm{s}}\right]\left[\frac{100 \mathrm{GeV}}{m_{\chi}}\right] \frac{\mathrm{erg}}{\mathrm{s}} .
$$

The luminosity of DM annihilation scales as $m_{\chi}^{-1}$, and has a large uncertainty associated to our limited knowledge of space dependence of the DM density, in particular near the galactic center. Note also that the emission is proportional to the square of the density and it is therefore enhanced if the DM is clumpy (since $\left\langle\rho_{\chi}^{2}\right\rangle \geq\left\langle\rho_{\chi}\right\rangle^{2}$ ).

The charged particles produced in the annihilations remain partially confined by the galactic magnetic fields inside the Galaxy, and are observable as an additional contribution to the fluxes of cosmic rays. The most sensitive obervations can be performed observing antiparticles, that is $e^{+}$and $\bar{p}$ because in this case the background from astrophysical processes is smaller.

The Pamela detector, in orbit since June 2006, and dedicated to the detection of cosmic rays has observed a large excess of positrons above $100 \mathrm{GeV}$ [65] that has generated an extraordinary interest. The positron excess was later confirmed by measurements of the Fermi detector [75] using the east-west effect to separate the electron and positron fluxes, and later with greater precision by the AMS detector [76-78].

Other detectors like Atic [79], Fermi [67] and Hess [80] that do not have the capability to separate positively and negatively charged particles have measured the sum of the $e^{ \pm}$spectra with results that are consistent with the existence of an additional source of $e^{ \pm}$(probably giving equal contributions to the $e^{+}$and $e^{-}$spectra).

The interpretation of these results has generated a large quantity of literature. Several authors have discussed the possibility that the "excess" of positrons (and possibly electrons) over the expected flux is due to the effect of DM annihilation. There are two main difficulties for this interpretation. The first is that one also expects an excess in the $\bar{p}$ flux, but this is not observed [64]. The relative yields of $e^{+}$and $\bar{p}$ in the self-annihilation of the DM particles are model dependent, but is very difficult to generate a large $e^{+}$signal without producing an observable effect for $\bar{p}$. To solve this problem one has to introduce some special ad hoc dynamics where for example the DM particles couple to leptons but not to quarks.

The second difficulty is that the size of the positron excess is much larger than expected, suggesting that the quantity $\langle\sigma v\rangle$ is larger than the cosmological prediction. A possible solution is to increase the average value $\left\langle\rho_{\chi}^{2}\right\rangle$ leaving $\left\langle\rho_{\chi}\right\rangle$ constant. This can be done introducing a "clumpiness" in the DM spatial distribution (since $\left\langle\rho_{\chi}^{2}\right\rangle \geq$ $\left\langle\rho_{\chi}\right\rangle^{2}$ ). Another possible solution is is to introduce some special dynamics so that $\langle\sigma v\rangle_{\mathrm{MW}}>\langle\sigma v\rangle_{\text {freeze out }}$ (the so called "Sommerfeld enhancement") making use of the fact that the velocity distribution of the DM particles in the Galaxy is lower than at the freeze out time

Important constraints on the existence of DM in the form of WIMPs have been obtained from photon observations. The photon flux coming from the direction $\Omega$ can be obtained integrating the emission over the line of sight:

$$
\phi_{\gamma}\left(E_{\gamma}, \Omega\right)=\left.\frac{\langle\sigma v\rangle}{2 m_{\chi}^{2}}\left[\int d \ell \rho^{2}(\ell, \Omega)\right] \frac{d N_{\gamma}}{d E_{\gamma}}\right|_{\chi \chi \rightarrow \gamma} .
$$

The observations of Fermi and of the Cherenkov telescopes have not given any evidence of the presence of a photon signal from WIMP annihilation (see Refs. [81-85]) setting important constraints to the DM interpretaton of the positron anomaly.

This is not the place for a complete discussion of the measurements of the positron and anti-proton fluxes and the possible interpretations. The main point that we want to make here is that a detailed understanding of hadronic interactions is important for the interpretations of the observations.

The reasons for an important relation between the search for a Dark Matter signal in positrons or antiprotons and the description of hadronic interactions can be outlined as follows. The annihilation of Dark Matter injects antiprotons (and similarly for positrons) in interstellar space at a rate:

$$
Q_{\bar{p}}^{\mathrm{DM}}(E)=\int d^{3} x \frac{\rho_{\chi}^{2}(\vec{x})}{m_{\chi}^{2}}\left\langle\sigma_{\chi \chi} v\right\rangle \frac{d N_{\chi \chi} \rightarrow \bar{p}}{d E}
$$

where the integral is over the Milky Way volume. A possible DM contribution must be disentangled from the background of particles created in the interactions of cosmic rays with interstellar gas, that inject an anti-proton rate:

$$
\begin{aligned}
Q_{\bar{p}}^{\mathrm{CR}}(E)= & \sum_{A} \int d^{3} x \int d E_{0} N_{A}\left(E_{0}, \vec{x}\right) \beta c n_{\mathrm{ism}}(\vec{x}) \\
& \times \frac{d \sigma_{A \rightarrow \bar{p}}}{d E}\left(E, E_{0}\right)
\end{aligned}
$$

where the sum is over all CR species ( $p$, and nuclei), and the cross section should be calculated averaging over the composition of the ISM medium (to a good approximation a combination of hydrogen and helium).

It is clear that a good knowledge of of the inclusive spectra of antiproton production in $p p, p-\mathrm{He}$, and $\mathrm{He}-$ $\mathrm{He}$ interactions is mandatory. A program of studies of these cross sections in accelerator experiments could be beneficial for an understanding of present and future observations.

\section{Outlook}

The study of the "high energy universe" continues to make rapid progress, thanks to an ensemble of new observatories 
that are producing a wealth of information using all three main "messengers", charged particles, gamma rays and neutrinos.

It is interesting to note that this has been historically a field "dominated" by the observers, starting with the completely unexpected discovery of cosmic rays a century ago and continuing with the detection of Pulsars, Active Galaxies, Gamma Ray Bursts, .... The string of surprises is continuing, with the Pamela positron anomaly, the Fermi bubbles, the IceCube high energy astrophysical neutrinos and so on. Serendipity seems to often play a crucial role, and theorists appear to be always a few steps behind, struggling to make sense of what they had not predicted.

It is possible that the study of high energy particles will play a crucial role in the understanding of the nature of Dark Matter. In fact if the "WIMP" idea is correct, this must be the case, and it will be essential to verify that the annihilation of DM particles in the Milky Way takes place with the cosmologically predicted rate, and to obtain measurements of the branching ratios into different final states. In this case, high energy astrophysics would become a fundamental arena for the study of the properties of the DM particles.

It is of course possible that the nature of the DM is completely different and is not connected to high energy particles. Also in this situation the "fundamental" interest of high energy, multi-messenger astrophysics is clear and strong, and it would be a serious naive error to dismiss the field as "just astrophysics".

To explore the open problems at the "boundaries of Science" we need new laboratories, that allow new observations for phenomena that are not yet accessible with the instruments and facilities available today. LHC is of course a wonderful example of such a new laboratory, but in any case, (whether or not new physics will be discovered in the future data) it will be necessary to go beyond.

The astrophysical sources that generate the high energy particles are potentially great laboratories to explore the validity of our physical laws, in conditions that cannot be reproduced on Earth. Only the Early Universe can provide a more extreme environment. It is therefore of great interest to understand the structure of these astrophysical objects and the physical processes that generate very high energy particles.

In this contribution we have tried to outline some of the relations between the studies of the high energy universe and of hadronic interactions. A more precise description of the properties of hadronic interaction is very important for the interpretation of the data of shower detectors that observe cosmic rays in the energy range $E \gtrsim 10 \mathrm{TeV}$.

The data collected at the high energy colliders, in particular at the LHC have been very important, to test and improve our understanding of hadronic interactions, but many questions remain open, and the uncertainties are still signficant for the interpretation of CR observations. More efforts in the direction of new and better measurements are very desirable and should be strongly supported by the community interested in CR science.

Measurements at lower energy can also be of very significant interest. It is obvious that to accurately describe a very high energy shower, one needs to model with precision, not only the first interaction of the primary particle, but also the many other interactions at lower energy. Even small uncertainties (of order 10-20\%) in the properties of lower energy collisions at $E \sim 10-1000 \mathrm{GeV}$ have non negligible effects on shower observables because of the large number of these interactions in a single shower.

Much of the information in this energy range has been obtained in experiments performed decades in the past. A well designed program of experimental studies in this energy range is also very desirable, and has the potential to obtain an important reduction of the uncertainties.

It should be stressed that understanding QCD in the non-perturbative regime should be seen as an important scientific task and a program of experimental studies about the properties of multiple particle production in hadronic interactions could stimulate theoretical studies bringing light to the "dark side" of the Standard Model.

As a final remark, we can recall that cosmic rays allow us to study hadronic interactions at c.m. energies much higher than attainable with accelerators, and it is also possible that valuable information about hadronic physics will be obtained using CR observations. In fact one can expect that in the future information will flow in both directions: from particle physics to astrophysics, and from astrophysics to particle physics.

I am very grateful to the organizers and participants of the Symposium for a very fruitful meeting. Special thanks are due to Ralph Engel, Tom Gaisser, Bryan Pattison and Silvia Vernetto.

\section{References}

[1] M. Tavani et al. [AGILE Collaboration], Astrophys. J. 710, L151 (2010) [arXiv:1001.5150 [astroph.HE]]

[2] M. Ackermann et al. [Fermi-LAT Collaboration], Science 339, 807 (2013) [arXiv:1302.3307 [astroph.HE]]

[3] Thomas Gaisser "Atmospheric lepton fluxes", in these proceeedings

[4] Emilio Radicioni, "Total and elastic cross sections in hadronic collisions", in these proceeedings

[5] K. Nakamura et al. [Particle Data Group Collaboration], J. Phys. G 37, 075021 (2010)

[6] J. Beringer et al. [Particle Data Group Collaboration], Phys. Rev. D 86, 010001 (2012)

[7] P. Abreu et al. [Pierre Auger Collaboration], Phys. Rev. Lett. 109, 062002 (2012) [arXiv:1208.1520 [hep-ex]]

[8] M. M. Block and F. Halzen, Phys. Rev. D 70, 091901 (2004) [hep-ph/0405174]

[9] L. V. Gribov, E. M. Levin and M. G. Ryskin, Phys. Rept. 100, 1 (1983)

[10] V. Barone and E. Predazzi, "High Energy Particle Diffraction", Springer-Verlag, Berlin (2002)

[11] Ralf Matthias Ulrich "Relation between hadronic interactions and air showers", in these proceeedings

[12] P. Lipari and M. Lusignoli, Phys. Rev. D 80, 074014 (2009) [arXiv:0908.0495 [hep-ph]] 
[13] P. Lipari and M. Lusignoli, Eur. Phys. J. C 73, 2630 (2013) [arXiv:1305.7216 [hep-ph]]

[14] Giulia Pancheri, "Models for photoproduction at ultra-high energies and photon initiated shower development", in these proceeedings

[15] R. P. Feynman, Phys. Rev. Lett. 23, 1415 (1969)

[16] R. D. Field and R. P. Feynman, Nucl. Phys. B 136, 1 (1978)

[17] B. Andersson, G. Gustafson, G. Ingelman and T. Sjostrand, Phys. Rept. 97, 31 (1983)

[18] T. Sjostrand, L. Lonnblad and S. Mrenna, hep-ph/0108264

[19] O. Adriani et al., Phys. Lett. B 703, 128 (2011) [arXiv:1104.5294 [hep-ex]]

[20] Yoshitaka Itow, "Forward hadron production at the LHC', in these proceeedings

[21] Hiroaki Menjo, "The recent results from LHCf", in these proceeedings

[22] T. Sjostrand and M. van Zijl, Phys. Rev. D 36, 2019 (1987).

[23] T. Sjostrand, S. Mrenna and P. Z. Skands, Comput. Phys. Commun. 178, 852 (2008) [arXiv:0710.3820 [hep-ph]]

[24] M. Bahr, S. Gieseke and M. H. Seymour, JHEP 0807, 076 (2008) [arXiv:0803.3633 [hep-ph]]

[25] T. Gleisberg et al. JHEP 0902, 007 (2009) [arXiv:0811.4622 [hep-ph]]

[26] Peter Skands "Modeling hadronic interactions in HEP MC generators", in these proceeedings

[27] N. N. Kalmykov and S. S. Ostapchenko, Phys. Atom. Nucl. 56, 346 (1993) [Yad. Fiz. 56N3, 105 (1993)]

[28] S. Ostapchenko, Phys. Rev. D 74, 014026 (2006) [hep-ph/0505259]

[29] E. J. Ahn, R. Engel, T. K. Gaisser, P. Lipari and T. Stanev, Phys. Rev. D 80, 094003 (2009) [arXiv:0906.4113 [hep-ph]]

[30] K. Werner, F. -M. Liu and T. Pierog, Phys. Rev. C 74, 044902 (2006) [hep-ph/0506232]

[31] Tanguy Pierog, "Modeling hadronic interactions in cosmic ray MC generators", in these proceeedings

[32] B. Alessandro et al., arXiv:1101.1852 [hep-ex]

[33] D. d'Enterria et al. Astropart. Phys. 35, 98 (2011) [arXiv:1101.5596 [astro-ph.HE]]

[34] Auger Coll., Phys. Rev. Lett. 104, 091101 (2010) [arXiv:1002.0699 [astro-ph.HE]]

[35] Auger Coll., Science 318, $938 \quad$ (2007) [arXiv:0711.2256 [astro-ph]]

[36] HiRes Coll., Phys. Rev. Lett. 104, 161101 (2010) [arXiv:0910.4184 [astro-ph.HE]]

[37] P. Sokolsky [TA and HiRes Collaboration], EPJ Web Conf. 52, 06002 (2013)

[38] [arXiv:1503.07540]

[39] Bruno Rossi \& Kenneth Greisen, Rev. Mod. Phys. 13, $240(1941)$

[40] W. Heitler "International Series of Monographs on Physics" Oxford: Clarendon (1954)

[41] James Matthews "Overview of UHECRs measurements", in these proceeedings

[42] Pierre Sokolsky "Recent Results from the Telescope Array Experiment", in these proceeedings
[43] Stanislav Knurenko "Mass Composition of Cosmic Rays at Ultra High Energies with Yakutsk Array.”, in these proceeedings

[44] H. S. Ahn et al., Astrophys. J. 714, L89-L93 (2010). [arXiv:1004.1123 [astro-ph.HE]]

[45] O. Adriani et al. [ Pamela Collaboration ], Science 332, 69-72 (2011). [arXiv:1103.4055 [astro-ph.HE]]

[46] M. Aguilar et al. (AMS Collaboration) Phys. Rev. Lett. 114, 171103 (2015)

[47] M. Amenomori [Tibet AS-gamma Collaboration], Science 314, 439-443 (2006) [astro-ph/0610 671]

[48] Milagro Coll., Astrophys. J. 698, 2121-2130 (2009). [arXiv:0806.2293 [astro-ph]]

[49] S. Vernetto et al., arXiv:0907.4615 [astro-ph.HE]

[50] IceCube Coll., Astrophys. J. 718, L194 (2010). [arXiv:1005.2960 [astro-ph.HE]]

[51] Milagro Coll., Phys. Rev. Lett. 101, 221101 (2008) [arXiv:0801.3827 [astro-ph]]

[52] L. Drury and F. Aharonian, Astropart. Phys. 29, 420 (2008) [arXiv:0802 . 4403 [astro-ph]]

[53] Kascade Coll., Astropart. Phys. 24, 1-25 (2005). [astro-ph/0505413]

[54] Kascade-Grande Coll., Phys. Rev. Lett. 107, 171104 (2011). [arXiv:1107.5885 [astro-ph.HE]]

[55] HiRes Coll., Phys. Rev. Lett. 100, 101101 (2008). [astro-ph/0703099]

[56] Auger Coll., Phys. Lett. B685, 239-246 (2010). [arXiv:1002.1975 [astro-ph.HE]]

[57] V. Berezinsky, A. Z. Gazizov \& S. I. Grigorieva, Phys. Lett. B 612, 147 (2005) [arXiv:astro-ph/0502550]

[58] D. R.Bergman, J. W. Belz, J. Phys. G G34, R359 (2007). [arXiv:0704.3721 [astro-ph]]

[59] P. Lipari, arXiv:0808.0417 [astro-ph]

[60] Auger Coll., Astropart. Phys. 34, 314 (2010) [arXiv:1009.1855 [astro-ph.HE]]

[61] Yakutsk collaboration, Pisma Zh. Eksp. Teor. Fiz. 87, 215 (2008) [JETP Lett. 87, 185 (2008)] [arXiv:0803.0612 [astro-ph]]

[62] R. U. Abbasi et al., arXiv:0804.0382 [astro-ph]

[63] J. Cronin, arXiv:astro-ph/0911.47141

[64] Pamela Coll., Phys. Rev. Lett. 102, 051101 (2009) [arXiv:0810.4994 [astro-ph]]

[65] Pamela Coll., Nature 458, 607 (2009) [arXiv:0810.4995 [astro-ph]]

[66] Pamela Coll., Phys. Rev. Lett. 106, 201101 (2011) [arXiv:1103.2880 [astro-ph.HE]]

[67] Fermi-LAT Coll., Phys. Rev. Lett. 102, 181101 (2009) [arXiv:0905.0025 [astro-ph.HE]]

[68] S. Profumo, Central Eur. J. Phys. 10, 1 (2011) [arXiv:0812.4457 [astro-ph]]

[69] D. Hooper, P. Blasi and P. D. Serpico, JCAP 0901, 025 (2009) [arXiv:0810.1527 [astro-ph]]

[70] Y.Z.Fan, B.Zhang, J.Chang, Int. J. Mod. Phys. D19, 2011-2058 (2010). [arXiv:1008.4646 [astroph.HE]]

[71] P. A. R. Ade et al. [Planck Collaboration], arXiv:1502.01589 [astro-ph.CO] 
[72] M. Milgrom, Astrophys. J. 270, 365 (1983)

[73] J. D. Bekenstein, Phys. Rev. D 70, 083509 (2004) [Phys. Rev. D 71, 069901 (2005)] [astro-ph/ 0403694]

[74] Jon Ellis, in these proceeedings

[75] Fermi LAT Coll., Phys. Rev. Lett. 108, 011103 (2012) [arXiv:1109. 0521 [astro-ph.HE]]

[76] M. Aguilar et al. [AMS Collaboration], Phys. Rev. Lett. 110, 141102 (2013)

[77] L. Accardo et al. [AMS Collaboration], Phys. Rev. Lett. 113, 121101 (2014)

[78] M. Aguilar et al. [AMS Collaboration], Phys. Rev. Lett. 113, 121102 (2014)
[79] ATIC Coll., Nature 456, 362-365 (2008)

[80] Hess Coll., Astron. Astrophys. 508, 561 (2009) [arXiv:0905.0105 [astro-ph.HE]]

[81] Fermi-LAT Coll., JCAP 1004, 014 (2010) [arXiv:1002.4415 [astro-ph.CO]]

[82] Fermi-LAT Coll., JCAP 1005, 025 (2010) [arXiv:1002 .2239 [astro-ph.CO]]

[83] Fermi-LAT Coll., Phys. Rev. Lett. 104, 091302 (2010) [arXiv:1001 . 4836 [astro-ph.HE]]

[84] Fermi-LAT Coll., Astrophys. J. 712, 147 (2010) [arXiv:1001.4531 [astro-ph.CO]]

[85] Fermi-LAT Coll., Phys. Rev. Lett. 107, 241302 (2011) [arXiv:1108.3546 [astro-ph.HE]] 